\title{
HIGH-DIMENSIONAL COVARIANCE MATRIX ESTIMATION IN APPROXIMATE FACTOR MODELS ${ }^{1}$
}

\author{
By JiAnQIng FAn, YuAn LiaO AND Martina MincheVA \\ Princeton University
}

\begin{abstract}
The variance-covariance matrix plays a central role in the inferential theories of high-dimensional factor models in finance and economics. Popular regularization methods of directly exploiting sparsity are not directly applicable to many financial problems. Classical methods of estimating the covariance matrices are based on the strict factor models, assuming independent idiosyncratic components. This assumption, however, is restrictive in practical applications. By assuming sparse error covariance matrix, we allow the presence of the cross-sectional correlation even after taking out common factors, and it enables us to combine the merits of both methods. We estimate the sparse covariance using the adaptive thresholding technique as in Cai and Liu [J. Amer. Statist. Assoc. 106 (2011) 672-684], taking into account the fact that direct observations of the idiosyncratic components are unavailable. The impact of high dimensionality on the covariance matrix estimation based on the factor structure is then studied.
\end{abstract}

1. Introduction. We consider a factor model defined as follows:

$$
y_{i t}=\mathbf{b}_{i}^{\prime} \mathbf{f}_{t}+u_{i t},
$$

where $y_{i t}$ is the observed datum for the $i$ th $(i=1, \ldots, p)$ asset at time $t=$ $1, \ldots, T ; \mathbf{b}_{i}$ is a $K \times 1$ vector of factor loadings; $\mathbf{f}_{t}$ is a $K \times 1$ vector of common factors, and $u_{i t}$ is the idiosyncratic error component of $y_{i t}$. Classical factor analysis assumes that both $p$ and $K$ are fixed, while $T$ is allowed to grow. However, in the recent decades, both economic and financial applications have encountered very large data sets which contain high-dimensional variables. For example, the World Bank has data for about two hundred countries over forty years; in portfolio allocation, the number of stocks can be in thousands and be larger or of the same order of the sample size. In modeling housing prices in each zip code, the number of regions can be of order thousands, yet the sample size can be 240 months or twenty years. The covariance matrix of order several thousands is critical for understanding the co-movement of housing prices indices over these zip codes.

\footnotetext{
Received May 2011; revised October 2011.

${ }^{1}$ Supported in part by NIH Grants R01-GM100474, R01-GM072611 and NSF Grant DMS-0704337.

MSC2010 subject classifications. Primary 62H25; secondary 62F12, 62H12.

Key words and phrases. Sparse estimation, thresholding, cross-sectional correlation, common factors, idiosyncratic, seemingly unrelated regression.
} 
Inferential theory of factor analysis relies on estimating $\boldsymbol{\Sigma}_{u}$, the variancecovariance matrix of the error term, and $\Sigma$, the variance-covariance matrix of $\mathbf{y}_{t}=\left(y_{1 t}, \ldots, y_{p t}\right)^{\prime}$. In the literature, $\boldsymbol{\Sigma}=\operatorname{cov}\left(\mathbf{y}_{t}\right)$ was traditionally estimated by the sample covariance matrix of $\mathbf{y}_{t}$.

$$
\Sigma_{\text {sam }}=\frac{1}{T-1} \sum_{t=1}^{T}\left(\mathbf{y}_{t}-\overline{\mathbf{y}}\right)\left(\mathbf{y}_{t}-\overline{\mathbf{y}}\right)^{\prime},
$$

which was always assumed to be pointwise root- $T$ consistent. However, the sample covariance matrix is an inappropriate estimator in high-dimensional settings. For example, when $p$ is larger than $T, \boldsymbol{\Sigma}_{\text {sam }}$ becomes singular while $\boldsymbol{\Sigma}$ is always strictly positive definite. Even if $p<T$, Fan, Fan and Lv (2008) showed that this estimator has a very slow convergence rate under the Frobenius norm. Realizing the limitation of the sample covariance estimator in high-dimensional factor models, Fan, Fan and Lv (2008) considered more refined estimation of $\boldsymbol{\Sigma}$, by incorporating the common factor structure. One of the key assumptions they made was the cross-sectional independence among the idiosyncratic components, which results in a diagonal matrix $\boldsymbol{\Sigma}_{u}=E \mathbf{u}_{t} \mathbf{u}_{t}^{\prime}$. The cross-sectional independence, however, is restrictive in many applications, as it rules out the approximate factor structure as in Chamberlain and Rothschild (1983). In this paper, we relax this assumption, and investigate the impact of the cross-sectional correlations of idiosyncratic noises on the estimation of $\boldsymbol{\Sigma}$ and $\boldsymbol{\Sigma}_{u}$, when both $p$ and $T$ are allowed to diverge. We show that the estimated covariance matrices are still invertible with probability approaching one, even if $p>T$. In particular, when estimating $\boldsymbol{\Sigma}^{-1}$ and $\boldsymbol{\Sigma}_{u}^{-1}$, we allow $p$ to increase much faster than $T$, say, $p=O\left(\exp \left(T^{\alpha}\right)\right)$, for some $\alpha \in(0,1)$.

Sparsity is one of the commonly used assumptions in the estimation of highdimensional covariance matrices, which assumes that many entries of the offdiagonal elements are zero, and the number of nonzero off-diagonal entries is restricted to grow slowly. Imposing the sparsity assumption directly on the covariance of $\mathbf{y}_{t}$, however, is inappropriate for many applications of finance and economics. In this paper we use the factor model and assume that $\boldsymbol{\Sigma}_{u}$ is sparse, and estimate both $\boldsymbol{\Sigma}_{u}$ and $\boldsymbol{\Sigma}_{u}^{-1}$ using the thresholding method [Bickel and Levina (2008a), Cai and Liu (2011)] based on the estimated residuals in the factor model. It is assumed that the factors $\mathbf{f}_{t}$ are observable, as in Fama and French (1992), Fan, Fan and Lv (2008), and many other empirical applications. We derive the convergence rates of both estimated $\boldsymbol{\Sigma}$ and its inverse, respectively, under various norms which are to be defined later. In addition, we achieve better convergence rates than those in Fan, Fan and Lv (2008).

Various approaches have been proposed to estimate a large covariance matrix: Bickel and Levina (2008a, 2008b) constructed the estimators based on regularization and thresholding, respectively. Rothman, Levina and Zhu (2009) considered thresholding the sample covariance matrix with more general thresholding functions. Lam and Fan (2009) proposed penalized quasi-likelihood method to achieve 
both the consistency and sparsistency of the estimation. More recently, Cai and Zhou (2010) derived the minimax rate for sparse matrix estimation, and showed that the thresholding estimator attains this optimal rate under the operator norm. Cai and Liu (2011) proposed a thresholding procedure which is adaptive to the variability of individual entries and unveiled its improved rate of convergence.

The rest of the paper is organized as follows. Section 2 provides the asymptotic theory for estimating the error covariance matrix and its inverse. Section 3 considers estimating the covariance matrix of $\mathbf{y}_{t}$. Section 4 extends the results to the seemingly unrelated regression model, a set of linear equations with correlated error terms in which the covariates are different across equations. Section 5 reports the simulation results. Finally, Section 6 concludes with discussions. All proofs are given in the Appendix. Throughout the paper, we use $\lambda_{\min }(\mathbf{A})$ and $\lambda_{\max }(\mathbf{A})$ to denote the minimum and maximum eigenvalues of a matrix $\mathbf{A}$. We also denote by $\|\mathbf{A}\|_{F},\|\mathbf{A}\|$ and $\|\mathbf{A}\|_{\text {MAX }}$ the Frobenius norm, operator norm and elementwise norm of a matrix $\mathbf{A}$, respectively, defined, respectively, as $\|\mathbf{A}\|_{F}=\operatorname{tr}^{1 / 2}\left(\mathbf{A}^{\prime} \mathbf{A}\right)$, $\|\mathbf{A}\|=\lambda_{\max }^{1 / 2}\left(\mathbf{A}^{\prime} \mathbf{A}\right)$ and $\|\mathbf{A}\|_{\text {MAX }}=\max _{i, j}\left|A_{i j}\right|$. Note that, when $\mathbf{A}$ is a vector, both $\|\mathbf{A}\|$ and $\|\mathbf{A}\|_{F}$ are equal to the Euclidean norm.

\section{Estimation of error covariance matrix.}

2.1. Adaptive thresholding. Consider the following approximate factor model, in which the cross-sectional correlation among the idiosyncratic error components is allowed:

$$
y_{i t}=\mathbf{b}_{i}^{\prime} \mathbf{f}_{t}+u_{i t},
$$

where $i=1, \ldots, p$ and $t=1, \ldots, T ; \mathbf{b}_{i}$ is a $K \times 1$ vector of factor loadings; $\mathbf{f}_{t}$ is a $K \times 1$ vector of observable common factors, uncorrelated with $u_{i t}$. Write

$$
\mathbf{B}=\left(\mathbf{b}_{1}, \ldots, \mathbf{b}_{p}\right)^{\prime}, \quad \mathbf{y}_{t}=\left(y_{1 t}, \ldots, y_{p t}\right)^{\prime} ; \quad \mathbf{u}_{t}=\left(u_{1 t}, \ldots, u_{p t}\right)^{\prime},
$$

then model (2.1) can be written in a more compact form,

$$
\mathbf{y}_{t}=\mathbf{B f}_{t}+\mathbf{u}_{t}
$$

with $E\left(\mathbf{u}_{t} \mid \mathbf{f}_{t}\right)=0$.

In practical applications, $p$ can be thought of as the number of assets or stocks, or number of regions in spatial and temporal problems such as home price indices or sales of drugs, and in practice can be of the same order as, or even larger than $T$. For example, an asset pricing model may contain hundreds of assets while the sample size on daily returns is less than several hundreds. In the estimation of the optimal portfolio allocation, it was observed by Fan, Fan and Lv (2008) that the effect of large $p$ on the convergence rate can be quite severe. In contrast, the number of common factors, $K$, can be much smaller. For example, the rank theory of consumer demand systems implies no more than three factors [e.g., Gorman (1981) and Lewbel (1991)]. 
The error covariance matrix

$$
\boldsymbol{\Sigma}_{u}=\operatorname{cov}\left(\mathbf{u}_{t}\right),
$$

itself is of interest for the inferential theory of factor models. For example, the asymptotic covariance of the least square estimator of $\mathbf{B}$ depends on $\boldsymbol{\Sigma}_{u}^{-1}$, and in simulating home price indices over a certain time horizon for mortgage based securities, a good estimate of $\Sigma_{u}$ is needed. When $p$ is close to or larger than $T$, estimating $\boldsymbol{\Sigma}_{u}$ is very challenging. Therefore, following the literature of highdimensional covariance matrix estimation, we assume it is sparse, that is, many of its off-diagonal entries are zeros. Specifically, let $\Sigma_{u}=\left(\sigma_{i j}\right)_{p \times p}$. Define

$$
m_{T}=\max _{i \leq p} \sum_{j \leq p} I\left(\sigma_{i j} \neq 0\right) .
$$

The sparsity assumption puts an upper bound restriction on $m_{T}$. Specifically, we assume

$$
m_{T}^{2}=o\left(\frac{T}{K^{2} \log p}\right) .
$$

In this formulation, we even allow the number of factors $K$ to be large, possibly growing with $T$.

A more general treatment [e.g., Bickel and Levina (2008a) and Cai and Liu (2011)] is to assume that the $l_{q}$ norm of the row vectors of $\boldsymbol{\Sigma}_{u}$ are uniformly bounded across rows by a slowly growing sequence, for some $q \in[0,1)$. In contrast, the assumption we make in this paper, that is, $q=0$, has clearer economic interpretation. For example, the firm returns can be modeled by the factor model, where $u_{i t}$ represents a firm's individual shock at time $t$. Driven by the industryspecific components, these shocks are correlated among the firms in the same industry, but can be assumed to be uncorrelated across industries, since the industryspecific components are not pervasive for the whole economy [Connor and Korajczyk (1993)].

We estimate $\boldsymbol{\Sigma}_{u}$ using the thresholding technique introduced and studied by Bickel and Levina (2008a), Rothman, Levina and Zhu (2009), Cai and Liu (2011), etc., which is summarized as follows: Suppose we observe data $\left(\mathbf{X}_{1}, \ldots, \mathbf{X}_{T}\right)$ of a $p \times 1$ vector $\mathbf{X}$, which follows a multivariate Gaussian distribution $N\left(0, \boldsymbol{\Sigma}_{X}\right)$. The sample covariance matrix of $\mathbf{X}$ is thus given by

$$
\mathbf{S}_{X}=\frac{1}{T} \sum_{i=1}^{T}\left(\mathbf{X}_{i}-\overline{\mathbf{X}}\right)\left(\mathbf{X}_{i}-\overline{\mathbf{X}}\right)^{\prime}=\left(s_{i j}\right)_{p \times p} .
$$

Define the thresholding operator by $\mathcal{T}_{t}(\mathbf{M})=\left(M_{i j} I\left(\left|M_{i j}\right| \geq t\right)\right)$ for any symmetric matrix M. Then $\mathcal{T}_{t}$ preserves the symmetry of $\mathbf{M}$. Let $\widehat{\boldsymbol{\Sigma}}_{X}^{\mathcal{T}}=\mathcal{T}_{\omega_{T}}\left(\mathbf{S}_{X}\right)$, where $\omega_{T}=$ $O(\sqrt{\log p / T})$. Bickel and Levina (2008a) then showed that

$$
\left\|\widehat{\boldsymbol{\Sigma}}_{X}^{\mathcal{T}}-\boldsymbol{\Sigma}_{X}\right\|=O_{p}\left(\omega_{T} m_{T}\right) .
$$


In the factor models, however, we do not observe the error term directly. Hence when estimating the error covariance matrix of a factor model, we need to construct a sample covariance matrix based on the residuals $\widehat{u}_{i t}$ before thresholding. The residuals are obtained using the plug-in method, by estimating the factor loadings first. Let $\widehat{\mathbf{b}}_{i}$ be the ordinary least square (OLS) estimator of $\mathbf{b}_{i}$, and

$$
\widehat{u}_{i t}=y_{i t}-\widehat{\mathbf{b}}_{i}^{\prime} \mathbf{f}_{t} .
$$

Denote by $\widehat{\mathbf{u}}_{t}=\left(\widehat{u}_{1 t}, \ldots, \widehat{u}_{p t}\right)^{\prime}$. We then construct the residual covariance matrix as

$$
\widehat{\boldsymbol{\Sigma}}_{u}=\frac{1}{T} \sum_{t=1}^{T} \widehat{\mathbf{u}}_{t} \widehat{\mathbf{u}}_{t}^{\prime}=\left(\widehat{\sigma}_{i j}\right) .
$$

Note that the thresholding value $\omega_{T}=O(\sqrt{\log p / T})$ in Bickel and Levina (2008a) is in fact obtained from the rate of convergence of $\max _{i j}\left|s_{i j}-\Sigma_{X, i j}\right|$. This rate changes when $s_{i j}$ is replaced with the residual $\widehat{u}_{i j}$, which will be slower if the number of common factors $K$ increases with $T$. Therefore, the thresholding value $\omega_{T}$ used in this paper is adjusted to account for the effect of the estimation of the residuals.

2.2. Asymptotic properties of the thresholding estimator. Bickel and Levina (2008a) used a universal constant as the thresholding value. As pointed out by Rothman, Levina and Zhu (2009) and Cai and Liu (2011), when the variances of the entries of the sample covariance matrix vary over a wide range, it is more desirable to use thresholds that capture the variability of individual estimation. For this purpose, in this paper, we apply the adaptive thresholding estimator [Cai and Liu (2011)] to estimate the error covariance matrix, which is given by

$$
\begin{aligned}
& \widehat{\Sigma}_{u}^{\mathcal{T}}=\left(\widehat{\sigma}_{i j}^{\mathcal{T}}\right), \quad \widehat{\sigma}_{i j}^{\mathcal{T}}=\widehat{\sigma}_{i j} I\left(\left|\widehat{\sigma}_{i j}\right| \geq \sqrt{\widehat{\theta}_{i j}} \omega_{T}\right), \\
& \widehat{\theta}_{i j}=\frac{1}{T} \sum_{t=1}^{T}\left(\widehat{u}_{i t} \widehat{u}_{j t}-\widehat{\sigma}_{i j}\right)^{2}
\end{aligned}
$$

for some $\omega_{T}$ to be specified later.

We impose the following assumptions:

ASSUMPTION 2.1. (i) $\left\{\mathbf{u}_{t}\right\}_{t \geq 1}$ is stationary and ergodic such that each $\mathbf{u}_{t}$ has zero mean vector and covariance matrix $\boldsymbol{\Sigma}_{u}$. In addition, the strong mixing condition in Assumption 3.2 holds.

(ii) There exist constants $c_{1}, c_{2}>0$ such that $c_{1}<\lambda_{\min }\left(\boldsymbol{\Sigma}_{u}\right) \leq \lambda_{\max }\left(\boldsymbol{\Sigma}_{u}\right)<c_{2}$, and $c_{1}<\operatorname{var}\left(u_{i t} u_{j t}\right)<c_{2}$ for all $i \leq p, j \leq p$.

(iii) There exist $r_{1}>0$ and $b_{1}>0$, such that for any $s>0$ and $i \leq p$,

$$
P\left(\left|u_{i t}\right|>s\right) \leq \exp \left(-\left(s / b_{1}\right)^{r_{1}}\right) .
$$


Condition (i) allows the idiosyncratic components to be weakly dependent. We will formally present the strong mixing condition in the next section. In order for the main results in this section to hold, it suffices to impose the strong mixing condition marginally on $\mathbf{u}_{t}$ only. Roughly speaking, we require the mixing coefficient

$$
\alpha(T)=\sup _{A \in \mathcal{F}_{-\infty}^{0}, B \in \mathcal{F}_{T}^{\infty}}|P(A) P(B)-P(A \cap B)|
$$

to decrease exponentially fast as $T \rightarrow \infty$, where $\left(\mathcal{F}_{-\infty}^{0}, \mathcal{F}_{T}^{\infty}\right)$ are the $\sigma$-algebras generated by $\left\{\mathbf{u}_{t}\right\}_{t=-\infty}^{0}$ and $\left\{\mathbf{u}_{t}\right\}_{t=T}^{\infty}$, respectively.

Condition (ii) requires the nonsingularity of $\boldsymbol{\Sigma}_{u}$. Note that Cai and Liu (2011) allowed $\max _{j} \sigma_{j j}$ to diverse when direct observations are available. Condition (ii), however, requires that $\sigma_{j j}$ should be uniformly bounded. In factor models, a uniform upper bound on the variance of $u_{i t}$ is needed when we estimate the covariance matrix of $\mathbf{y}_{t}$ later. This assumption is satisfied by most of the applications of factor models. Condition (iii) requires the distributions of $\left(u_{1 t}, \ldots, u_{p t}\right)$ to have exponential-type tails, which allows us to apply the large deviation theory to $\frac{1}{T} \sum_{t=1}^{T} u_{i t} u_{j t}-\sigma_{i j}$.

AsSUMPTION 2.2. There exist positive sequences $\kappa_{1}(p, T)=o(1), \kappa_{2}(p$, $T)=o(1)$ and $a_{T}=o(1)$, and a constant $M>0$, such that for all $C>M$,

$$
\begin{array}{r}
P\left(\max _{i \leq p} \frac{1}{T} \sum_{t=1}^{T}\left|u_{i t}-\widehat{u}_{i t}\right|^{2}>C a_{T}^{2}\right) \leq O\left(\kappa_{1}(p, T)\right), \\
P\left(\max _{i \leq p, t \leq T}\left|u_{i t}-\widehat{u}_{i t}\right|>C\right) \leq O\left(\kappa_{2}(p, T)\right) .
\end{array}
$$

This assumption allows us to apply thresholding to the estimated error covariance matrix when direct observations are not available, without introducing too much extra estimation error. Note that it permits a general case when the original "data" is contaminated, including any type of estimate of the data when direct observations are not available, as well as the case when data is subject to measurement of errors. We will show in the next section that in a linear factor model when $\left\{u_{i t}\right\}_{i \leq p, t \leq T}$ are estimated using the OLS estimator, the rate of convergence $a_{T}^{2}=\left(K^{2} \log p\right) / T$.

The following theorem establishes the asymptotic properties of the thresholding estimator $\widehat{\boldsymbol{\Sigma}}_{u}^{\mathcal{T}}$, based on observations with estimation errors. Let $\gamma^{-1}=3 r_{1}^{-1}+$ $r_{2}^{-1}$, where $r_{1}$ and $r_{2}$ are defined in Assumptions 2.1, 3.2, respectively.

THEOREM 2.1. Suppose $\gamma<1$ and $(\log p)^{6 / \gamma-1}=o(T)$. Then under Assumptions 2.1 and 2.2, there exist $C_{1}>0$ and $C_{2}>0$ such that for $\widehat{\Sigma}_{u}^{\mathcal{T}}$ defined in (2.5) with

$$
\omega_{T}=C_{1}\left(\sqrt{\frac{\log p}{T}}+a_{T}\right)
$$


we have

$$
P\left(\left\|\widehat{\boldsymbol{\Sigma}}_{u}^{\mathcal{T}}-\boldsymbol{\Sigma}_{u}\right\| \leq C_{2} \omega_{T} m_{T}\right) \geq 1-O\left(\frac{1}{p^{2}}+\kappa_{1}(p, T)+\kappa_{2}(p, T)\right) .
$$

In addition, if $\omega_{T} m_{T}=o(1)$, then with probability at least $1-O\left(\frac{1}{p^{2}}+\kappa_{1}(p, T)+\right.$ $\left.\kappa_{2}(p, T)\right)$,

$$
\lambda_{\min }\left(\widehat{\boldsymbol{\Sigma}}_{u}^{\mathcal{T}}\right) \geq 0.5 \lambda_{\min }\left(\boldsymbol{\Sigma}_{u}\right)
$$

and

$$
\left\|\left(\widehat{\boldsymbol{\Sigma}}_{u}^{\mathcal{T}}\right)^{-1}-\boldsymbol{\Sigma}_{u}^{-1}\right\| \leq C_{2} \omega_{T} m_{T}
$$

Note that we derive result (2.7) without assuming the sparsity on $\boldsymbol{\Sigma}_{u}$, that is, no restriction is imposed on $m_{T}$. When $\omega_{T} m_{T} \neq o(1),(2.7)$ still holds, but $\| \widehat{\boldsymbol{\Sigma}}_{u}^{\mathcal{T}}-$ $\Sigma_{u} \|$ does not converge to zero in probability. On the other hand, the condition $\omega_{T} m_{T}=o(1)$ is required to preserve the nonsingularity of $\widehat{\boldsymbol{\Sigma}}_{u}^{\mathcal{T}}$ asymptotically and to consistently estimate $\Sigma_{u}^{-1}$.

The rate of convergence also depends on the averaged estimation error of the residual terms. We will see in the next section that when the number of common factors $K$ increases slowly, the convergence rate in Theorem 2.1 is close to the minimax optimal rate as in Cai and Zhou (2010).

3. Estimation of covariance matrix using factors. We now investigate the estimation of the covariance matrix $\boldsymbol{\Sigma}$ in the approximate factor model

$$
\mathbf{y}_{t}=\mathbf{B} \mathbf{f}_{t}+\mathbf{u}_{t},
$$

where $\Sigma=\operatorname{cov}\left(\mathbf{y}_{t}\right)$. This covariance matrix is particularly of interest in many applications of factor models as well as corresponding inferential theories. When estimating a large dimensional covariance matrix, sparsity and banding are two commonly used assumptions for regularization [e.g., Bickel and Levina (2008a, 2008b)]. In most of the applications in finance and economics, however, these two assumptions are inappropriate for $\boldsymbol{\Sigma}$. For instance, the US housing prices in the county level are generally associated with a few national indices, and there is no natural ordering among the counties. Hence neither the sparsity nor the banding is realistic for such a problem. On the other hand, it is natural to assume $\boldsymbol{\Sigma}_{u}$ sparse, after controlling the common factors. Therefore, our approach combines the merits of both the sparsity and factor structures.

Note that

$$
\boldsymbol{\Sigma}=\mathbf{B} \operatorname{cov}\left(\mathbf{f}_{t}\right) \mathbf{B}^{\prime}+\boldsymbol{\Sigma}_{u} .
$$

By the Sherman-Morrison-Woodbury formula,

$$
\boldsymbol{\Sigma}^{-1}=\boldsymbol{\Sigma}_{u}^{-1}-\boldsymbol{\Sigma}_{u}^{-1} \mathbf{B}\left[\operatorname{cov}\left(\mathbf{f}_{t}\right)^{-1}+\mathbf{B}^{\prime} \boldsymbol{\Sigma}_{u}^{-1} \mathbf{B}\right]^{-1} \mathbf{B}^{\prime} \boldsymbol{\Sigma}_{u}^{-1} .
$$


When the factors are observable, one can estimate $\mathbf{B}$ by the least squares method, $\widehat{\mathbf{B}}=\left(\widehat{\mathbf{b}}_{1}, \ldots, \widehat{\mathbf{b}}_{p}\right)^{\prime}$, where

$$
\widehat{\mathbf{b}}_{i}=\arg \min _{\mathbf{b}_{i}} \frac{1}{T p} \sum_{t=1}^{T} \sum_{i=1}^{p}\left(y_{i t}-\mathbf{b}_{i}^{\prime} \mathbf{f}_{t}\right)^{2} .
$$

The covariance matrix $\operatorname{cov}\left(\mathbf{f}_{t}\right)$ can be estimated by the sample covariance matrix

$$
\widehat{\operatorname{cov}}\left(\mathbf{f}_{t}\right)=T^{-1} \mathbf{X} \mathbf{X}^{\prime}-T^{-2} \mathbf{X} 11^{\prime} \mathbf{X}^{\prime}
$$

where $\mathbf{X}=\left(\mathbf{f}_{1}, \ldots, \mathbf{f}_{T}\right)$, and $\mathbf{1}$ is a $T$-dimensional column vector of ones. Therefore, by employing the thresholding estimator $\widehat{\boldsymbol{\Sigma}}_{u}^{\mathcal{T}}$ in (2.5), we obtain substitution estimators

$$
\widehat{\boldsymbol{\Sigma}}^{\mathcal{T}}=\widehat{\mathbf{B}} \widehat{\operatorname{cov}}\left(\mathbf{f}_{t}\right) \widehat{\mathbf{B}}^{\prime}+\widehat{\boldsymbol{\Sigma}}_{u}^{\mathcal{T}}
$$

and

$$
\left.\left(\widehat{\Sigma}^{\mathcal{T}}\right)^{-1}=\left(\widehat{\boldsymbol{\Sigma}}_{u}^{\mathcal{T}}\right)^{-1}-\left(\widehat{\boldsymbol{\Sigma}}_{u}^{\mathcal{T}}\right)^{-1} \widehat{\mathbf{B}} \widehat{\operatorname{cov}}\left(\mathbf{f}_{t}\right)^{-1}+\widehat{\mathbf{B}}^{\prime}\left(\widehat{\boldsymbol{\Sigma}}_{u}^{\mathcal{T}}\right)^{-1} \widehat{\mathbf{B}}\right]^{-1} \widehat{\mathbf{B}}^{\prime}\left(\widehat{\boldsymbol{\Sigma}}_{u}^{\mathcal{T}}\right)^{-1}
$$

In practice, one may apply a common thresholding $\lambda$ to the correlation matrix of $\widehat{\Sigma}_{u}$, and then use the substitution estimator similar to (3.1). When $\lambda=0$ (no thresholding), the resulting estimator is the sample covariance, whereas when $\lambda=1$ (all off-diagonals are thresholded), the resulting estimator is an estimator based on the strict factor model [Fan, Fan and Lv (2008)]. Thus we have created a path (indexed by $\lambda$ ) which connects the nonparametric estimate of covariance matrix to the parametric estimate.

The following assumptions are made:

Assumption 3.1. (i) $\left\{\mathbf{f}_{t}\right\}_{t \geq 1}$ is stationary and ergodic.

(ii) $\left\{\mathbf{u}_{t}\right\}_{t \geq 1}$ and $\left\{\mathbf{f}_{t}\right\}_{t \geq 1}$ are independent.

In addition to the conditions above, we introduce the strong mixing conditions to conduct asymptotic analysis of the least square estimates. Let $\mathcal{F}_{-\infty}^{0}$ and $\mathcal{F}_{T}^{\infty}$ denote the $\sigma$-algebras generated by $\left\{\left(\mathbf{f}_{t}, \mathbf{u}_{t}\right):-\infty \leq t \leq 0\right\}$ and $\left\{\left(\mathbf{f}_{t}, \mathbf{u}_{t}\right): T \leq t \leq\right.$ $\infty$ \}, respectively. In addition, define the mixing coefficient

$$
\alpha(T)=\sup _{A \in \mathcal{F}_{-\infty}^{0}, B \in \mathcal{F}_{T}^{\infty}}|P(A) P(B)-P(A B)| .
$$

The following strong mixing assumption enables us to apply the Bernstein's inequality in the technical proofs.

Assumption 3.2. There exist positive constants $r_{2}$ and $C$ such that for all $t \in \mathbb{Z}^{+}$

$$
\alpha(t) \leq \exp \left(-C t^{r_{2}}\right)
$$


In addition, we impose the following regularity conditions:

Assumption 3.3. (i) There exists a constant $M>0$ such that for all $i, j$ and $t, E y_{i t}^{2}<M, E f_{i t}^{2}<M$ and $\left|b_{i j}\right|<M$.

(ii) There exists a constant $r_{3}>0$ with $3 r_{3}^{-1}+r_{2}^{-1}>1$, and $b_{2}>0$ such that for any $s>0$ and $i \leq K$,

$$
P\left(\left|f_{i t}\right|>s\right) \leq \exp \left(-\left(s / b_{2}\right)^{r_{3}}\right) .
$$

Condition (ii) allows us to apply the Bernstein-type inequality for the weakly dependent data.

Assumption 3.4. There exists a constant $C>0$ such that $\lambda_{\min }\left(\operatorname{cov}\left(\mathbf{f}_{t}\right)\right)>C$.

Assumptions 3.4 and 2.1 ensure that both $\lambda_{\min }\left(\operatorname{cov}\left(\mathbf{f}_{t}\right)\right)$ and $\lambda_{\min }(\boldsymbol{\Sigma})$ are bounded away from zero, which is needed to derive the convergence rate of $\left\|\left(\widehat{\boldsymbol{\Sigma}}^{\mathcal{T}}\right)^{-1}-\boldsymbol{\Sigma}^{-1}\right\|$ below.

The following lemma verifies Assumption 2.2, which derives the rate of convergence of the OLS estimator as well as the estimated residuals.

Let $\gamma_{2}^{-1}=1.5 r_{1}^{-1}+1.5 r_{3}^{-1}+r_{2}^{-1}$.

LEMmA 3.1. Suppose $K=o(p), K^{4}(\log p)^{2}=o(T)$ and $(\log p)^{2 / \gamma_{2}-1}=$ $o(T)$. Then under the assumptions of Theorem 2.1 and Assumptions 3.1-3.4, there exists $C>0$, such that:

(i)

$$
P\left(\max _{i \leq p}\left\|\widehat{\mathbf{b}}_{i}-\mathbf{b}_{i}\right\|>C \sqrt{\frac{K \log p}{T}}\right)=O\left(\frac{1}{p^{2}}+\frac{1}{T^{2}}\right) ;
$$

(ii)

$$
P\left(\max _{i \leq p} \frac{1}{T} \sum_{t=1}^{T}\left|u_{i t}-\widehat{u}_{i t}\right|^{2}>\frac{C K^{2} \log p}{T}\right)=O\left(\frac{1}{p^{2}}+\frac{1}{T^{2}}\right)
$$

(iii)

$$
P\left(\max _{i \leq p, t \leq T}\left|u_{i t}-\widehat{u}_{i t}\right|>C K(\log T)^{1 / r_{3}} \sqrt{\frac{\log p}{T}}\right)=O\left(\frac{1}{p^{2}}+\frac{1}{T^{2}}\right) .
$$

By Lemma 3.1 and Assumption 2.2, $a_{T}=K \sqrt{(\log p) / T}$ and $\kappa_{1}(p, T)=$ $\kappa_{2}(p, T)=p^{-2}+T^{-2}$. Therefore in the linear approximate factor model, the thresholding parameter $\omega_{T}$ defined in Theorem 2.1 is simplified to the following: for some positive constant $C_{1}^{\prime}$,

$$
\omega_{T}=C_{1}^{\prime} K \sqrt{\frac{\log p}{T}} .
$$


Now we can apply Theorem 2.1 to obtain the following theorem:

THEOREM 3.1. Under the assumptions of Lemma 3.1, there exist $C_{1}^{\prime}>0$ and $C_{2}^{\prime}>0$ such that the adaptive thresholding estimator defined in (2.5) with $\omega_{T}^{2}=$ $C_{1}^{\prime} \frac{K^{2} \log p}{T}$ satisfies:

(i)

$$
P\left(\left\|\widehat{\boldsymbol{\Sigma}}_{u}^{\mathcal{T}}-\boldsymbol{\Sigma}_{u}\right\| \leq C_{2}^{\prime} m_{T} K \sqrt{\frac{\log p}{T}}\right)=1-O\left(\frac{1}{p^{2}}+\frac{1}{T^{2}}\right) .
$$

(ii) If $m_{T} K \sqrt{\frac{\log p}{T}}=o(1)$, then with probability at least $1-O\left(\frac{1}{p^{2}}+\frac{1}{T^{2}}\right)$,

$$
\lambda_{\min }\left(\widehat{\Sigma}_{u}^{\mathcal{T}}\right) \geq 0.5 \lambda_{\min }\left(\boldsymbol{\Sigma}_{u}\right)
$$

and

$$
\left\|\left(\widehat{\Sigma}_{u}^{\mathcal{T}}\right)^{-1}-\Sigma_{u}^{-1}\right\| \leq C_{2}^{\prime} m_{T} K \sqrt{\frac{\log p}{T}} .
$$

REMARK 3.1. We briefly comment on the terms in the convergence rate above.

(1) The term $K$ appears as an effect of using the estimated residuals to construct the thresholding covariance estimator, which is typically small compared to $p$ and $T$ in many applications. For instance, the famous Fama-French three-factor model shows that $K=3$ factors are adequate for the US equity market. In an empirical study on asset returns, Bai and $\mathrm{Ng}$ (2002) used the monthly data which contains the returns of 4883 stocks for sixty months. For their data set, $T=60, p=4883$. Bai and $\mathrm{Ng}$ (2002) determined $K=2$ common factors.

(2) As in Bickel and Levina (2008a) and Cai and Liu (2011), $m_{T}$, the maximum number of nonzero components across the rows of $\boldsymbol{\Sigma}_{u}$, also plays a role in the convergence rate. Note that when $K$ is bounded, the convergence rate reduces to $O_{p}\left(m_{T} \sqrt{(\log p) / T}\right)$, the same as the minimax rate derived by Cai and Zhou (2010).

One of our main objectives is to estimate $\boldsymbol{\Sigma}$, which is the $p \times p$ dimensional covarinace matrix of $\mathbf{y}_{t}$, assumed to be time invariant. We can achieve a better accuracy in estimating both $\boldsymbol{\Sigma}$ and $\boldsymbol{\Sigma}^{-1}$ by incorporating the factor structure than using the sample covariance matrix, as shown by Fan, Fan and Lv (2008) in the strict factor model case. When the cross-sectional correlations among the idiosyncratic components $\left(u_{1 t}, \ldots, u_{p t}\right)$ are in presence, we can still take advantage of the factor structure. This is particularly essential when direct sparsity assumption on $\boldsymbol{\Sigma}$ is inappropriate. 
Assumption 3.5. $\left\|p^{-1} \mathbf{B}^{\prime} \mathbf{B}-\boldsymbol{\Omega}\right\|=o(1)$ for some $K \times K$ symmetric positive definite matrix $\boldsymbol{\Omega}$ such that $\lambda_{\min }(\boldsymbol{\Omega})$ is bounded away from zero.

Assumption 3.5 requires that the factors should be pervasive, that is, impact every individual time series [Harding (2009)]. It was imposed by Fan, Fan and Lv (2008) only when they tried to establish the asymptotic normality of the covariance estimator. However, it turns out to be also helpful to obtain a good upper bound of $\left\|\left(\widehat{\boldsymbol{\Sigma}}^{\mathcal{T}}\right)^{-1}-\boldsymbol{\Sigma}^{-1}\right\|$, as it ensures that $\lambda_{\max }\left(\left(\mathbf{B}^{\prime} \boldsymbol{\Sigma}^{-1} \mathbf{B}\right)^{-1}\right)=O\left(p^{-1}\right)$.

Fan, Fan and Lv (2008) obtained an upper bound of $\left\|\widehat{\boldsymbol{\Sigma}}^{\mathcal{T}}-\boldsymbol{\Sigma}\right\|_{F}$ under the Frobenius norm when $\boldsymbol{\Sigma}_{u}$ is diagonal, that is, there was no cross-sectional correlation among the idiosyncratic errors. In order for their upper bound to decrease to zero, $p^{2}<T$ is required. Even with this restrictive assumption, they showed that the convergence rate is the same as the usual sample covariance matrix of $\mathbf{y}_{t}$, though the latter does not take the factor structure into account. Alternatively, they considered the entropy loss norm, proposed by James and Stein (1961),

$$
\left\|\widehat{\boldsymbol{\Sigma}}^{\mathcal{T}}-\boldsymbol{\Sigma}\right\|_{\Sigma}=\left(p^{-1} \operatorname{tr}\left[\left(\widehat{\boldsymbol{\Sigma}}^{\mathcal{T}} \boldsymbol{\Sigma}^{-1}-I\right)^{2}\right]\right)^{1 / 2}=p^{-1 / 2}\left\|\boldsymbol{\Sigma}^{-1 / 2}\left(\widehat{\boldsymbol{\Sigma}}^{\mathcal{T}}-\boldsymbol{\Sigma}\right) \boldsymbol{\Sigma}^{-1 / 2}\right\|_{F} .
$$

Here the factor $p^{-1 / 2}$ is used for normalization, such that $\|\Sigma\|_{\Sigma}=1$. Under this norm, Fan, Fan and Lv (2008) showed that the substitution estimator has a better convergence rate than the usual sample covariance matrix. Note that the normalization factor $p^{-1 / 2}$ in the definition results in an averaged estimation error, which also cancels out the diverging dimensionality introduced by $p$. In addition, for any two $p \times p$ matrices $\mathbf{A}_{1}$ and $\mathbf{A}_{2}$,

$$
\begin{aligned}
\left\|\mathbf{A}_{1}-\mathbf{A}_{2}\right\|_{\Sigma} & =p^{-1 / 2}\left\|\boldsymbol{\Sigma}^{-1 / 2}\left(\mathbf{A}_{1}-\mathbf{A}_{2}\right) \boldsymbol{\Sigma}^{-1 / 2}\right\|_{F} \\
& \leq\left\|\boldsymbol{\Sigma}^{-1 / 2}\left(\mathbf{A}_{1}-\mathbf{A}_{2}\right) \boldsymbol{\Sigma}^{-1 / 2}\right\| \\
& \leq\left\|\mathbf{A}_{1}-\mathbf{A}_{2}\right\| \cdot \lambda_{\max }\left(\boldsymbol{\Sigma}^{-1}\right) .
\end{aligned}
$$

Combining with the estimated low-rank matrix $\mathbf{B} \operatorname{cov}\left(\mathbf{f}_{t}\right) \mathbf{B}^{\prime}$, Theorem 3.1 implies the main theorem in this section:

THEOREM 3.2. Suppose $\log T=o(p)$. Under the assumptions of Theorem 3.1 and Assumption 3.5, we have:

(i)

$$
\begin{aligned}
& P\left(\left\|\widehat{\boldsymbol{\Sigma}}^{\mathcal{T}}-\Sigma\right\|_{\Sigma}^{2} \leq \frac{C p K^{2}(\log p)^{2}}{T^{2}}+\frac{C m_{T}^{2} K^{2} \log p}{T}\right) \\
& \quad=1-O\left(\frac{1}{p^{2}}+\frac{1}{T^{2}}\right), \\
& P\left(\left\|\widehat{\boldsymbol{\Sigma}}^{\mathcal{T}}-\Sigma\right\|_{\mathrm{MAX}}^{2} \leq \frac{C K^{2} \log p+C K^{4} \log T}{T}\right) \\
& =1-O\left(\frac{1}{p^{2}}+\frac{1}{T^{2}}\right) .
\end{aligned}
$$


(ii) If $m_{T} K \sqrt{\frac{\log p}{T}}=o(1)$, with probability at least $1-O\left(\frac{1}{p^{2}}+\frac{1}{T^{2}}\right)$,

$$
\lambda_{\min }\left(\widehat{\boldsymbol{\Sigma}}^{\mathcal{T}}\right) \geq 0.5 \lambda_{\min }\left(\boldsymbol{\Sigma}_{u}\right)
$$

and

$$
\left\|\left(\widehat{\Sigma}^{\mathcal{T}}\right)^{-1}-\Sigma^{-1}\right\| \leq C m_{T} K \sqrt{\frac{\log p}{T}} .
$$

Note that we have derived a better convergence rate of $\left(\widehat{\Sigma}^{\mathcal{T}}\right)^{-1}$ than that in Fan, Fan and Lv (2008). When the operator norm is considered, $p$ is allowed to grow exponentially fast in $T$ in order for $\left(\widehat{\Sigma}^{\mathcal{T}}\right)^{-1}$ to be consistent.

We have also derived the maximum elementwise estimation $\left\|\widehat{\boldsymbol{\Sigma}}^{\mathcal{T}}-\boldsymbol{\Sigma}\right\|_{\text {MAX }}$. This quantity appears in risk assessment as in Fan, Zhang and Yu (2008). For any portfolio with allocation vector $\mathbf{w}$, the true portfolio variance and the estimated one are given by $\mathbf{w}^{\prime} \boldsymbol{\Sigma} \mathbf{w}$ and $\mathbf{w}^{\prime} \widehat{\boldsymbol{\Sigma}}^{\mathcal{T}} \mathbf{w}$, respectively. The estimation error is bounded by

$$
\left|\mathbf{w}^{\prime} \widehat{\boldsymbol{\Sigma}}^{\mathcal{T}} \mathbf{w}-\mathbf{w}^{\prime} \mathbf{\Sigma} \mathbf{w}\right| \leq\left\|\widehat{\boldsymbol{\Sigma}}^{\mathcal{T}}-\boldsymbol{\Sigma}\right\|_{\operatorname{MAX}}\|\mathbf{w}\|_{1}^{2},
$$

where $\|\mathbf{w}\|_{1}$, the $l_{1}$ norm of $\mathbf{w}$, is the gross exposure of the portfolio.

4. Extension: Seemingly unrelated regression. A seemingly unrelated regression model [Kmenta and Gilbert (1970)] is a set of linear equations in which the disturbances are correlated across equations. Specifically, we have

$$
y_{i t}=\mathbf{b}_{i}^{\prime} \mathbf{f}_{i t}+u_{i t}, \quad i \leq p, t \leq T,
$$

where $\mathbf{b}_{i}$ and $\mathbf{f}_{i t}$ are both $K_{i} \times 1$ vectors. The $p$ linear equations (4.1) are related because their error terms $u_{i t}$ are correlated; that is, the covariance matrix

$$
\Sigma_{u}=\left(E u_{i t} u_{j t}\right)_{p \times p}
$$

is not diagonal.

Model (4.1) allows each variable $y_{i t}$ to have its own factors. This is important for many applications. In financial applications, the returns of individual stock depend on common market factors and sector-specific factors. In housing price index modeling, housing price appreciations depend on both national factors and local economy. When $\mathbf{f}_{i t}=\mathbf{f}_{t}$ for each $i \leq p$, model (4.1) reduces to the approximate factor model (1.1) with common factors $\mathbf{f}_{t}$.

Under mild conditions, running OLS on each equation produces unbiased and consistent estimator of $\mathbf{b}_{i}$ separately. However, since OLS does not take into account the cross-sectional correlation among the noises, it is not efficient. Instead, statisticians obtain the best linear unbiased estimator (BLUE) via generalized least square (GLS). Write

$$
\begin{aligned}
& \mathbf{y}_{i}=\left(y_{i 1}, \ldots, y_{i T}\right)^{\prime}, T \times 1, \quad \mathbf{X}_{i}=\left(\mathbf{f}_{i 1}, \ldots, \mathbf{f}_{i T}\right)^{\prime}, T \times K_{i}, \quad i \leq p, \\
& \mathbf{y}=\left(\begin{array}{c}
\mathbf{y}_{1} \\
\vdots \\
\mathbf{y}_{p}
\end{array}\right), \quad \mathbf{X}=\left(\begin{array}{ccc}
\mathbf{X}_{1} & 0 & 0 \\
0 & \ddots & 0 \\
0 & 0 & \mathbf{X}_{p}
\end{array}\right), \quad \mathbf{B}=\left(\begin{array}{c}
\mathbf{b}_{1} \\
\vdots \\
\mathbf{b}_{p}
\end{array}\right) .
\end{aligned}
$$


The GLS estimator of B is given by Zellner (1962).

$$
\widehat{\mathbf{B}}_{\mathrm{GLS}}=\left[\mathbf{X}^{\prime}\left(\widehat{\boldsymbol{\Sigma}}_{u}^{-1} \otimes I_{T}\right)^{-1} \mathbf{X}\right]^{-1}\left[\mathbf{X}^{\prime}\left(\widehat{\boldsymbol{\Sigma}}_{u}^{-1} \otimes I_{T}\right)^{-1} \mathbf{y}\right],
$$

where $I_{T}$ denotes a $T \times T$ identity matrix, $\otimes$ represents the Kronecker product operation and $\widehat{\boldsymbol{\Sigma}}_{u}$ is a consistent estimator of $\boldsymbol{\Sigma}_{u}$.

In classical seemingly unrelated regression in which $p$ does not grow with $T$, $\Sigma_{u}$ is estimated by a two-stage procedure [Kmenta and Gilbert (1970)]: In the first stage, estimate $\mathbf{B}$ via OLS, and obtain residuals

$$
\widehat{u}_{i t}=y_{i t}-\widehat{\mathbf{b}}_{i}^{\prime} \mathbf{f}_{i t} \text {. }
$$

In the second stage, estimate $\boldsymbol{\Sigma}_{u}$ by

$$
\widehat{\mathbf{\Sigma}}_{u}=\left(\widehat{\sigma}_{i j}\right)=\left(\frac{1}{T} \sum_{t=1}^{T} \widehat{u}_{i t} \widehat{u}_{j t}\right)_{p \times p} .
$$

In high dimensional, seemingly unrelated regression in which $p>T$, however, $\widehat{\boldsymbol{\Sigma}}_{u}$ is not invertible, and hence the GLS estimator (4.2) is infeasible.

By the sparsity assumption of $\boldsymbol{\Sigma}_{u}$, we can deal with this singularity problem by using the adaptive thresholding estimator, and produce a consistent nonsingular estimator of $\boldsymbol{\Sigma}_{u}$,

$$
\widehat{\boldsymbol{\Sigma}}_{u}^{\mathcal{T}}=\left(\widehat{\sigma}_{i j} I\left(\left|\widehat{\sigma}_{i j}\right|>\sqrt{\widehat{\theta}_{i j}} \omega_{T}\right)\right), \quad \widehat{\theta}_{i j}=\frac{1}{T} \sum_{t=1}^{T}\left(\widehat{u}_{i t} \widehat{u}_{j t}-\widehat{\sigma}_{i j}\right)^{2} .
$$

To pursue this goal, we impose the following assumptions:

ASSUMPTION 4.1. For each $i \leq p$ :

(i) $\left\{\mathbf{f}_{i t}\right\}_{t \geq 1}$ is stationary and ergodic.

(ii) $\left\{\mathbf{u}_{t}\right\}_{t \geq 1}$ and $\left\{\mathbf{f}_{i t}\right\}_{t \geq 1}$ are independent.

ASSUMPTION 4.2. There exists positive constants $C$ and $r_{2}$ such that for each $i \leq p$, the strong mixing condition

$$
\alpha(t) \leq \exp \left(-C t^{r_{2}}\right)
$$

is satisfied by $\left(\mathbf{f}_{i t}, \mathbf{u}_{t}\right)$.

ASSUMPTION 4.3. There exist constants $M$ and $C>0$ such that for all $i \leq$ $p, j \leq K_{i}, t \leq T$ :

(i) $E y_{i t}^{2}<M,\left|b_{i j}\right|<M$ and $E f_{i t, j}^{2}<M$.

(ii) $\min _{i \leq p} \lambda_{\min }\left(\operatorname{cov}\left(\mathbf{f}_{i t}\right)\right)>C$. 
Assumption 4.4. There exists a constant $r_{4}>0$ with $3 r_{4}^{-1}+r_{2}^{-1}>1$, and $b_{3}>0$ such that for any $s>0$ and $i, j$,

$$
P\left(\left|f_{i t, j}\right|>s\right) \leq \exp \left(-\left(s / b_{3}\right)^{r_{4}}\right) .
$$

These assumptions are similar to those made in Section 3, except that here they are imposed on the sector-specific factors. The main theorem in this section is a direct application of Theorem 2.1, which shows that the adaptive thresholding produces a consistent nonsingular estimator of $\widehat{\boldsymbol{\Sigma}}_{u}$.

THEOREM 4.1. Let $K=\max _{i \leq p} K_{i}$ and $\gamma_{3}^{-1}=1.5 r_{1}^{-1}+1.5 r_{4}^{-1}+r_{2}^{-1}$; suppose $K=o(p), K^{4}(\log p)^{2}=o(T)$ and $(\log p)^{2 / \gamma_{3}-1}=o(T)$. Under Assumptions 2.1, 4.1-4.4, there exist constants $C_{1}>0$ and $C_{2}>0$ such that the adaptive thresholding estimator defined in (4.5) with $\omega_{T}^{2}=C_{1} \frac{K^{2} \log p}{T}$ satisfies:

(i)

$$
P\left(\left\|\widehat{\boldsymbol{\Sigma}}_{u}^{\mathcal{T}}-\Sigma_{u}\right\| \leq C_{2} m_{T} K \sqrt{\frac{\log p}{T}}\right)=1-O\left(\frac{1}{p^{2}}+\frac{1}{T^{2}}\right)
$$

(ii) If $m_{T} K \sqrt{\frac{\log p}{T}}=o(1)$, then with probability at least $1-O\left(\frac{1}{p^{2}}+\frac{1}{T^{2}}\right)$,

$$
\lambda_{\min }\left(\widehat{\Sigma}_{u}^{\mathcal{T}}\right) \geq 0.5 \lambda_{\min }\left(\Sigma_{u}\right)
$$

and

$$
\left\|\left(\widehat{\Sigma}_{u}^{\mathcal{T}}\right)^{-1}-\Sigma_{u}^{-1}\right\| \leq C_{2} m_{T} K \sqrt{\frac{\log p}{T}} .
$$

Therefore, in the case when $p>T$, Theorem 4.1 enables us to efficiently estimate $\mathbf{B}$ via feasible GLS.

$$
\widehat{\mathbf{B}}_{\mathrm{GLS}}^{\mathcal{T}}=\left[\mathbf{X}^{\prime}\left(\left(\widehat{\boldsymbol{\Sigma}}_{u}^{\mathcal{T}}\right)^{-1} \otimes I_{T}\right)^{-1} \mathbf{X}\right]^{-1}\left[\mathbf{X}^{\prime}\left(\left(\widehat{\boldsymbol{\Sigma}}_{u}^{\mathcal{T}}\right)^{-1} \otimes I_{T}\right)^{-1} \mathbf{y}\right] .
$$

5. Monte Carlo experiments. In this section, we use simulation to demonstrate the rates of convergence of the estimators $\widehat{\boldsymbol{\Sigma}}^{\mathcal{T}}$ and $\left(\widehat{\boldsymbol{\Sigma}}^{\mathcal{T}}\right)^{-1}$ that we have obtained so far. The simulation model is a modified version of the Fama-French three-factor model described in Fan, Fan and Lv (2008). We fix the number of factors, $K=3$, and the length of time, $T=500$, and let the dimensionality $p$ gradually increase.

The Fama-French three-factor model [Fama and French (1992)] is given by

$$
y_{i t}=b_{i 1} f_{1 t}+b_{i 2} f_{2 t}+b_{i 3} f_{3 t}+u_{i t},
$$

which models the excess return (real rate of return minus risk-free rate) of the $i$ th stock of a portfolio, $y_{i t}$, with respect to 3 factors. The first factor is the excess 
return of the whole stock market, and the weighted excess return on all NASDAQ, AMEX and NYSE stocks is a commonly used proxy. It extends the capital assets pricing model (CAPM) by adding two new factors-SMB ("small minus big" cap) and HML ("high minus low" book/price). These two were added to the model after the observation that two types of stocks - small caps, and high book value to price ratio- tend to outperform the stock market as a whole.

We separate this section into three parts, calibration, simulation and results. Similarly to Section 5 of Fan, Fan and Lv (2008), in the calibration part we want to calculate realistic multivariate distributions from which we can generate the factor loadings $\mathbf{B}$, idiosyncratic noises $\left\{\mathbf{u}_{t}\right\}_{t=1}^{T}$ and the observable factors $\left\{\mathbf{f}_{t}\right\}_{t=1}^{T}$. The data was obtained from the data library of Kenneth French's website.

5.1. Calibration. To estimate the parameters in the Fama-French model, we will use the two-year daily data $\left(\tilde{\mathbf{y}}_{t}, \tilde{\mathbf{f}}_{t}\right)$ from Jan 1 st, 2009 to Dec 31st, 2010 ( $T=$ 500) of 30 industry portfolios.

(1) Calculate the least squares estimator $\tilde{\mathbf{B}}$ of $\tilde{\mathbf{y}}_{t}=\mathbf{B} \tilde{\mathbf{f}}_{t}+\mathbf{u}_{t}$, and take the rows of $\tilde{\mathbf{B}}$, namely $\tilde{\mathbf{b}}_{1}=\left(b_{11}, b_{12}, b_{13}\right), \ldots, \tilde{\mathbf{b}}_{30}=\left(b_{30,1}, b_{30,2}, b_{30,3}\right)$, to calculate the sample mean vector $\boldsymbol{\mu}_{B}$ and sample covariance matrix $\boldsymbol{\Sigma}_{B}$. The results are depicted in Table 1 . We then create a mutlivariate normal distribution $N_{3}\left(\boldsymbol{\mu}_{B}, \boldsymbol{\Sigma}_{B}\right)$, from which the factor loadings $\left\{\mathbf{b}_{i}\right\}_{i=1}^{p}$ are drawn.

(2) For each fixed $p$, create the sparse matrix $\boldsymbol{\Sigma}_{u}=\mathbf{D}+\mathbf{s s}^{\prime}-\operatorname{diag}\left\{s_{1}^{2}, \ldots, s_{p}^{2}\right\}$ in the following way. Let $\widehat{\mathbf{u}}_{t}=\tilde{\mathbf{y}}_{t}-\tilde{\mathbf{B}}_{t}$. For $i=1, \ldots, 30$, let $\widehat{\sigma}_{i}$ denote the standard deviation of the residuals of the $i$ th portfolio. We find $\min \left(\widehat{\sigma}_{i}\right)=0.3533$, $\max \left(\widehat{\sigma}_{i}\right)=1.5222$ and calculate the mean and the standard deviation of the $\widehat{\sigma}_{i}$ 's, namely $\bar{\sigma}=0.6055$ and $\sigma_{\mathrm{SD}}=0.2621$.

Let $\mathbf{D}=\operatorname{diag}\left\{\sigma_{1}^{2}, \ldots, \sigma_{p}^{2}\right\}$, where $\sigma_{1}, \ldots, \sigma_{p}$ are generated independently from the Gamma distribution $G(\alpha, \beta)$, with mean $\alpha \beta$ and standard deviation $\alpha^{1 / 2} \beta$. We match these values to $\bar{\sigma}=0.6055$ and $\sigma_{\mathrm{SD}}=0.2621$, to get $\alpha=5.6840$ and $\beta=0.1503$. Further, we create a loop that only accepts the value of $\sigma_{i}$ if it is between $\min \left(\widehat{\sigma}_{i}\right)=0.3533$ and $\max \left(\widehat{\sigma}_{i}\right)=1.5222$.

Create $\mathbf{s}=\left(s_{1}, \ldots, s_{p}\right)^{\prime}$ to be a sparse vector. We set each $s_{i} \sim N(0,1)$ with probability $\frac{0.2}{\sqrt{p} \log p}$, and $s_{i}=0$ otherwise. This leads to an average of $\frac{0.2 \sqrt{p}}{\log p}$ nonzero elements per each row of the error covariance matrix.

TABLE 1

Mean and covariance matrix used to generate $\mathbf{b}$

\begin{tabular}{llll}
\hline $\boldsymbol{\mu}_{\boldsymbol{B}}$ & \multicolumn{3}{c}{$\boldsymbol{\Sigma}_{\boldsymbol{B}}$} \\
\hline 1.0641 & 0.0475 & 0.0218 & 0.0488 \\
0.1233 & 0.0218 & 0.0945 & 0.0215 \\
-0.0119 & 0.0488 & 0.0215 & 0.1261 \\
\hline
\end{tabular}


TABLE 2

Parameters of $\mathbf{f}_{t}$ generating process

\begin{tabular}{|c|c|c|c|c|c|c|}
\hline$\mu$ & \multicolumn{3}{|c|}{$\operatorname{cov}\left(\mathbf{f}_{t}\right)$} & \multicolumn{3}{|c|}{$\Phi$} \\
\hline 0.1074 & 2.2540 & 0.2735 & 0.9197 & -0.1149 & 0.0024 & 0.0776 \\
\hline 0.0357 & 0.2735 & 0.3767 & 0.0430 & 0.0016 & -0.0162 & 0.0387 \\
\hline 0.0033 & 0.9197 & 0.0430 & 0.6822 & -0.0399 & 0.0218 & 0.0351 \\
\hline
\end{tabular}

Create a loop that generates $\Sigma_{u}$ multiple times until it is positive definite.

(3) Assume the factors follow the vector autoregressive [VAR(1)] model $\mathbf{f}_{t}=$ $\boldsymbol{\mu}+\boldsymbol{\Phi} \mathbf{f}_{t-1}+\boldsymbol{\varepsilon}_{t}$ for some $3 \times 3$ matrix $\boldsymbol{\Phi}$, where $\boldsymbol{\varepsilon}_{t}$ 's are i.i.d. $N_{3}\left(0, \boldsymbol{\Sigma}_{\epsilon}\right)$. We estimate $\boldsymbol{\Phi}, \boldsymbol{\mu}$ and $\boldsymbol{\Sigma}_{\epsilon}$ from the data, and obtain $\operatorname{cov}\left(\mathbf{f}_{t}\right)$. They are summarized in Table 2.

5.2. Simulation. For each fixed $p$, we generate $\left(\mathbf{b}_{1}, \ldots, \mathbf{b}_{p}\right)$ independently from $N_{3}\left(\boldsymbol{\mu}_{B}, \boldsymbol{\Sigma}_{B}\right)$, and generate $\left\{\mathbf{f}_{t}\right\}_{t=1}^{T}$ and $\left\{\mathbf{u}_{t}\right\}_{t=1}^{T}$ independently. We keep $T=$ 500 fixed, and gradually increase $p$ from 20 to 600 in multiples of 20 to illustrate the rates of convergence when the number of variables diverges with respect to the sample size.

Repeat the following steps $N=200$ times for each fixed $p$ : $\left.\mathbf{b}_{p}\right)^{\prime}$.

(1) Generate $\left\{\mathbf{b}_{i}\right\}_{i=1}^{p}$ independently from $N_{3}\left(\boldsymbol{\mu}_{B}, \boldsymbol{\Sigma}_{B}\right)$, and set $\mathbf{B}=\left(\mathbf{b}_{1}, \ldots\right.$,

(2) Generate $\left\{\mathbf{u}_{t}\right\}_{t=1}^{T}$ independently from $N_{p}\left(0, \boldsymbol{\Sigma}_{u}\right)$.

(3) Generate $\left\{\mathbf{f}_{t}\right\}_{t=1}^{T}$ independently from the $\operatorname{VAR}(1)$ model $\mathbf{f}_{t}=\boldsymbol{\mu}+\boldsymbol{\Phi} \mathbf{f}_{t-1}+$ $\boldsymbol{\varepsilon}_{t}$.

(4) Calculate $\mathbf{y}_{t}=\mathbf{B} \mathbf{f}_{t}+\mathbf{u}_{t}$ for $t=1, \ldots, T$.

(5) Set $\omega_{T}=0.10 K \sqrt{\log p / T}$ to obtain the thresholding estimator (2.5) $\widehat{\boldsymbol{\Sigma}}_{u}^{\mathcal{T}}$ and the sample covariance matrices $\widehat{\operatorname{cov}}\left(\mathbf{f}_{t}\right), \widehat{\boldsymbol{\Sigma}}_{y}=\frac{1}{T-1} \sum_{t=1}^{T}\left(\mathbf{y}_{t}-\overline{\mathbf{y}}\right)\left(\mathbf{y}_{t}-\overline{\mathbf{y}}\right)^{T}$.

We graph the convergence of $\widehat{\boldsymbol{\Sigma}}^{\mathcal{T}}$ and $\widehat{\boldsymbol{\Sigma}}_{y}$ to $\boldsymbol{\Sigma}$, the covariance matrix of $\mathbf{y}$, under the entropy-loss norm $\|\cdot\|_{\Sigma}$ and the elementwise norm $\|\cdot\|_{\text {MAX }}$. We also graph the convergence of the inverses $\left(\widehat{\Sigma}^{\mathcal{T}}\right)^{-1}$ and $\widehat{\Sigma}_{y}^{-1}$ to $\Sigma^{-1}$ under the operator norm. Note that we graph that only for $p$ from 20 to 300. Since $T=500$, for $p>500$ the sample covariance matrix is singular. Also, for $p$ close to $500, \widehat{\boldsymbol{\Sigma}}_{y}$ is nearly singular, which leads to abnormally large values of the operator norm. Last, we record the standard deviations of these norms.

5.3. Results. In Figures 1-3, the dashed curves correspond to $\widehat{\Sigma}^{\mathcal{T}}$ and the solid curves correspond to the sample covariance matrix $\widehat{\boldsymbol{\Sigma}}_{y}$. Figures 1 and 2 present the averages and standard deviations of the estimation error of both of these matrices with respect to the $\Sigma$-norm and infinity norm, respectively. Figure 3 presents the 

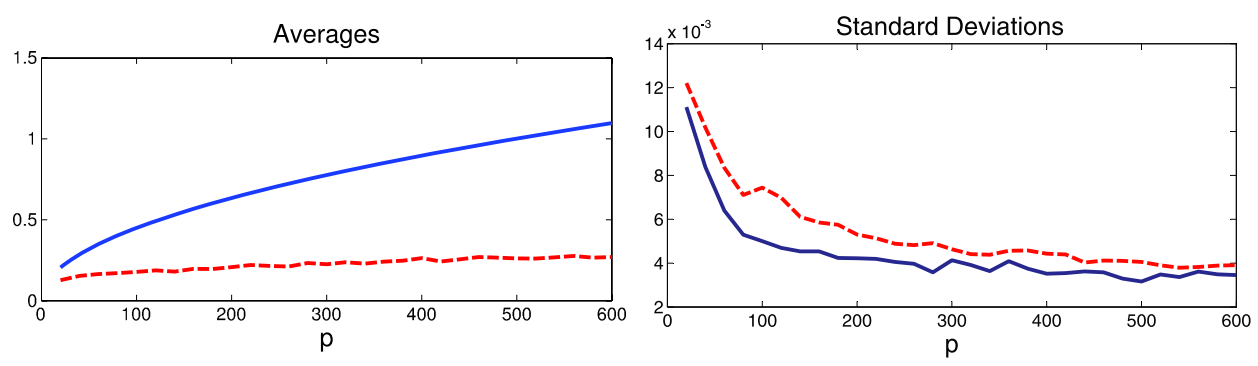

FIG. 1. Averages and standard deviations of $\left\|\widehat{\boldsymbol{\Sigma}}^{\mathcal{T}}-\boldsymbol{\Sigma}\right\|_{\Sigma}$ (dashed curve) and $\left\|\widehat{\boldsymbol{\Sigma}}_{y}-\boldsymbol{\Sigma}\right\|_{\Sigma}$ (solid curve) over $N=200$ iterations, as a function of the dimensionality $p$.

averages and estimation errors of the inverses with respect to the operator norm. Based on the simulation results, we can make the following observations:

(1) The standard deviations of the norms are negligible when compared to their corresponding averages.

(2) Under the $\|\cdot\|_{\Sigma}$, our estimate of the covariance matrix of $\mathbf{y}, \widehat{\Sigma}^{\mathcal{T}}$ performs much better than the sample covaraince matrix $\widehat{\Sigma}_{y}$. Note that, in the proof of Theorem 2 in Fan, Fan and Lv (2008), it was shown that

$$
\left\|\widehat{\boldsymbol{\Sigma}}_{y}-\boldsymbol{\Sigma}\right\|_{\Sigma}^{2}=O_{p}\left(\frac{K^{3}}{T p}\right)+O_{p}\left(\frac{p}{T}\right)+O_{p}\left(\frac{K^{3 / 2}}{T}\right) .
$$

For a small fixed value of $K$, such as $K=3$, the dominating term in (5.1) is $O\left(\frac{p}{T}\right)$. From Theorem 4.1, and given that $m_{T}=o\left(p^{1 / 4}\right)$, the dominating term in the convergence of $\left\|\widehat{\boldsymbol{\Sigma}}^{\mathcal{T}}-\boldsymbol{\Sigma}\right\|_{\Sigma}^{2}$ is $O_{p}\left(\frac{p}{T^{2}}+\frac{m_{T}^{2} \log p}{T}\right)$. So, we would expect our estimator to perform better, and the simulation results are consistent with the theory.

(3) Under the infinity norm, both estimators perform roughly the same. This is to be expected, given that the thresholding affects mainly the elements of the covariance matrix that are closest to 0 , and the infinity norm depicts the magnitude of the largest elementwise absolute error.
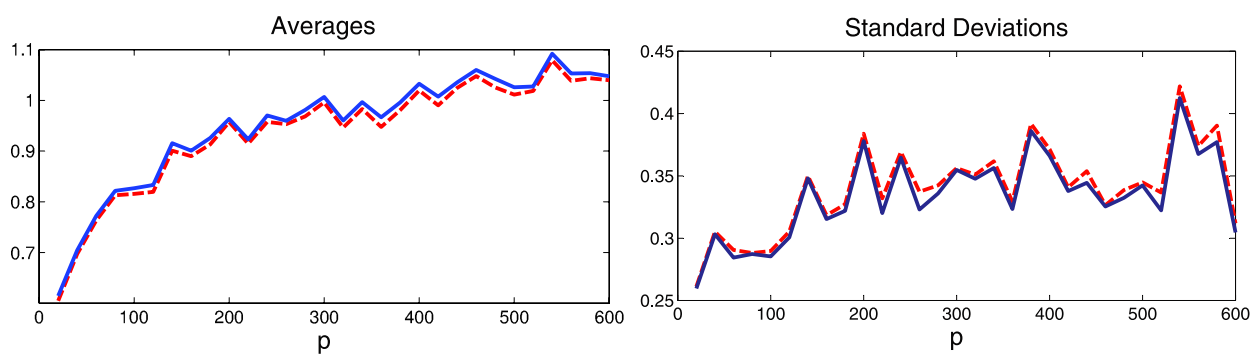

FIG. 2. Averages and standard deviations of $\left\|\widehat{\boldsymbol{\Sigma}}^{\mathcal{T}}-\boldsymbol{\Sigma}\right\|_{\mathrm{MAX}}$ (dashed curve) and $\left\|\widehat{\boldsymbol{\Sigma}}_{y}-\boldsymbol{\Sigma}\right\|_{\mathrm{MAX}}$ (solid curve) over $N=200$ iterations, as a function of the dimensionality $p$. 

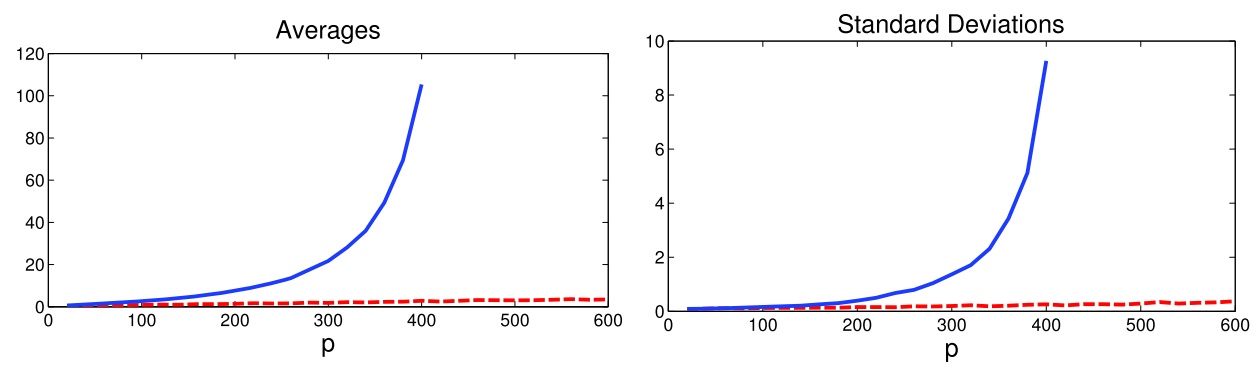

FIG. 3. Averages and standard deviations of $\left\|\left(\widehat{\boldsymbol{\Sigma}}^{\mathcal{T}}\right)^{-1}-\boldsymbol{\Sigma}^{-1}\right\|$ (dashed curve) and $\left\|\widehat{\boldsymbol{\Sigma}}_{y}^{-1}-\boldsymbol{\Sigma}^{-1}\right\|$ (solid curve) over $N=200$ iterations, as a function of the dimensionality $p$.

(4) Under the operator norm, the inverse of our estimator, $\left(\widehat{\boldsymbol{\Sigma}}^{\mathcal{T}}\right)^{-1}$ also performs significantly better than the inverse of the sample covariance matrix.

(5) Finally, when $p>500$, the thresholding estimators $\widehat{\boldsymbol{\Sigma}}_{u}^{\mathcal{T}}$ and $\widehat{\boldsymbol{\Sigma}}^{\mathcal{T}}$ are still nonsingular.

In conclusion, even after imposing less restrictive assumptions on the error covariance matrix, we still reach an estimator $\widehat{\boldsymbol{\Sigma}}^{\mathcal{T}}$ that significantly outperforms the standard sample covariance matrix.

6. Conclusions and discussions. We studied the rate of convergence of highdimensional covariance matrix of approximate factor models under various norms. By assuming sparse error covariance matrix, we allow for the presence of the cross-sectional correlation even after taking out common factors. Since direct observations of the noises are not available, we constructed the error sample covariance matrix, first based on the estimation residuals, and then estimated the error covariance matrix using the adaptive thresholding method. We then constructed the covariance matrix of $\mathbf{y}_{t}$ using the factor model, assuming that the factors follow a stationary and ergodic process, but can be weakly dependent. It was shown that after thresholding, the estimated covariance matrices are still invertible even if $p>T$, and the rate of convergence of $\left(\widehat{\boldsymbol{\Sigma}}^{\mathcal{T}}\right)^{-1}$ and $\left(\widehat{\boldsymbol{\Sigma}}_{u}^{\mathcal{T}}\right)^{-1}$ is of order $O_{p}\left(K m_{T} \sqrt{\log p / T}\right)$, where $K$ comes from the impact of estimating the unobservable noise terms. This demonstrates when estimating the inverse covariance matrix, $p$ is allowed to be much larger than $T$.

In fact, the rate of convergence in Theorem 2.1 reflects the impact of unobservable idiosyncratic components on the thresholding method. Generally, whether it is the minimax rate when direct observations are not available but have to be estimated is an important question, which is left as a research direction in the future.

Moreover, this paper uses the hard-thresholding technique, which takes the form of $\widehat{\sigma}_{i j}\left(\sigma_{i j}\right)=\sigma_{i j} I\left(\left|\sigma_{i j}\right|>\theta_{i j}\right)$ for some pre-determined threshold $\theta_{i j}$. Recently, Rothman, Levina and Zhu (2009) and Cai and Liu (2011) studied a more general thresholding function of Antoniadis and Fan (2001), which admits the form 
$\widehat{\sigma}_{i j}\left(\theta_{i j}\right)=s\left(\sigma_{i j}\right)$, and also allows for soft-thresholding. It is easy to apply the more general thresholding here as well, and the rate of convergence of the resulting covariance matrix estimators should be straightforward to derive.

Finally, we considered the case when common factors are observable, as in Fama and French (1992). In some applications, the common factors are unobservable and need to be estimated [Bai (2003)]. In that case, it is still possible to consistently estimate the covariance matrices using similar techniques as those in this paper. However, the impact of high dimensionality on the rate of convergence comes also from the estimation error of the unobservable factors. We plan to address this problem in a separate paper.

\section{APPENDIX A: PROOFS FOR SECTION 2}

A.1. Lemmas. The following lemmas are useful to be proved first, in which we consider the operator norm $\|\mathbf{A}\|^{2}=\lambda_{\max }\left(\mathbf{A}^{\prime} \mathbf{A}\right)$.

Lemma A.1. Let $\mathbf{A}$ be an $m \times m$ random matrix, $\mathbf{B}$ be an $m \times m$ deterministic matrix and both $\mathbf{A}$ and $\mathbf{B}$ are semi-positive definite. If there exists a positive sequence $\left\{c_{T}\right\}_{T=1}^{\infty}$ such that for all large enough $T, \lambda_{\min }(\mathbf{B})>c_{T}$. Then

$$
P\left(\lambda_{\min }(\mathbf{A}) \geq 0.5 c_{T}\right) \geq P\left(\|\mathbf{A}-\mathbf{B}\| \leq 0.5 c_{T}\right)
$$

and

$$
P\left(\left\|\mathbf{A}^{-1}-\mathbf{B}^{-1}\right\| \leq \frac{2}{c_{T}^{2}}\|\mathbf{A}-\mathbf{B}\|\right) \geq P\left(\|\mathbf{A}-\mathbf{B}\| \leq 0.5 c_{T}\right) .
$$

Proof. For any $\mathbf{v} \in \mathbb{R}^{m}$ such that $\|\mathbf{v}\|=1$, under the event $\|\mathbf{A}-\mathbf{B}\| \leq 0.5 c_{T}$,

$$
\begin{aligned}
\mathbf{v}^{\prime} \mathbf{A} \mathbf{v} & =\mathbf{v}^{\prime} \mathbf{B v}-\mathbf{v}^{\prime}(\mathbf{B}-\mathbf{A}) \mathbf{v} \geq \lambda_{\min }(\mathbf{B})-\|\mathbf{A}-\mathbf{B}\| \\
& \geq 0.5 c_{T} .
\end{aligned}
$$

Hence $\lambda_{\min }(\mathbf{A}) \geq 0.5 c_{T}$.

In addition, still under the event $\|\mathbf{A}-\mathbf{B}\| \leq 0.5 c_{T}$,

$$
\begin{aligned}
\left\|\mathbf{A}^{-1}-\mathbf{B}^{-1}\right\| & =\left\|\mathbf{A}^{-1}(\mathbf{B}-\mathbf{A}) \mathbf{B}^{-1}\right\| \\
& \leq \lambda_{\min }(\mathbf{A})^{-1}\|\mathbf{A}-\mathbf{B}\| \lambda_{\min }(\mathbf{B})^{-1} \\
& =2 c_{T}^{-1}\|\mathbf{A}-\mathbf{B}\| .
\end{aligned}
$$

LEMmA A.2. Suppose that the random variables $Z_{1}, Z_{2}$ both satisfy the exponential-type tail condition: There exist $r_{1}, r_{2} \in(0,1)$ and $b_{1}, b_{2}>0$, such that $\forall s>0$,

$$
P\left(\left|Z_{i}\right|>s\right) \leq \exp \left(1-\left(s / b_{i}\right)^{r_{i}}\right), \quad i=1,2 .
$$

Then for some $r_{3}$ and $b_{3}>0$, and any $s>0$,

$$
P\left(\left|Z_{1} Z_{2}\right|>s\right) \leq \exp \left(1-\left(s / b_{3}\right)^{r_{3}}\right) .
$$


PROOF. We have, for any $s>0, M=\left(s b_{2}^{r_{2} / r_{1}} / b_{1}\right)^{r_{1} /\left(r_{1}+r_{2}\right)}, b=b_{1} b_{2}$ and $r=r_{1} r_{2} /\left(r_{1}+r_{2}\right)$,

$$
\begin{aligned}
P\left(\left|Z_{1} Z_{2}\right|>s\right) & \leq P\left(M\left|Z_{1}\right|>s\right)+P\left(\left|Z_{2}\right|>M\right) \\
& \leq \exp \left(1-\left(s / b_{1} M\right)^{r_{1}}\right)+\exp \left(1-\left(M / b_{2}\right)^{r_{2}}\right) \\
& =2 \exp \left(1-(s / b)^{r}\right) .
\end{aligned}
$$

Pick up an $r_{3} \in(0, r)$, and $b_{3}>\max \left\{\left(r_{3} / r\right)^{1 / r} b,(1+\log 2)^{1 / r} b\right\}$; then it can be shown that $F(s)=(s / b)^{r}-\left(s / b_{3}\right)^{r_{3}}$ is increasing when $s>b_{3}$. Hence $F(s)>$ $F\left(b_{3}\right)>\log 2$ when $s>b_{3}$, which implies when $s>b_{3}$,

$$
P\left(\left|Z_{1} Z_{2}\right|>s\right) \leq 2 \exp \left(1-(s / b)^{r}\right) \leq \exp \left(1-\left(s / b_{3}\right)^{r_{3}}\right) .
$$

When $s \leq b_{3}$,

$$
P\left(\left|Z_{1} Z_{2}\right|>s\right) \leq 1 \leq \exp \left(1-\left(s / b_{3}\right)^{r_{3}}\right) .
$$

LEMMA A.3. Under the assumptions of Theorem 2.1, there exists a constant $C_{r}>0$ that does not depend on $(p, T)$, such that when $C>C_{r}$,

(i)

$$
P\left(\max _{i, j \leq p}\left|\frac{1}{T} \sum_{t=1}^{T} u_{i t} u_{j t}-\sigma_{i j}\right|>C \sqrt{\frac{\log p}{T}}\right)=O\left(\frac{1}{p^{2}}\right)
$$

(ii)

$$
P\left(\max _{i, j \leq p}\left|\frac{1}{T} \sum_{t=1}^{T}\left(\widehat{u}_{i t} \widehat{u}_{j t}-u_{i t} u_{j t}\right)\right|>C a_{T}\right)=O\left(\frac{1}{p^{2}}+\kappa_{1}(p, T)\right),
$$

(iii)

$$
P\left(\max _{i, j \leq p}\left|\widehat{\sigma}_{i j}-\sigma_{i j}\right|>C\left(\sqrt{\frac{\log p}{T}}+a_{T}\right)\right)=O\left(\frac{1}{p^{2}}+\kappa_{1}(p, T)\right) .
$$

ProOF. (i) By Assumption 2.1 and Lemma A.2, $u_{i t} u_{j t}$ satisfies the exponential tail condition, with parameter $r_{1} / 3$ as shown in the proof of Lemma A.2. Therefore by the Bernstein's inequality [Theorem 1 of Merlevède, Peligrad and Rio (2009)], there exist constants $C_{1}, C_{2}, C_{3}, C_{4}$ and $C_{5}>0$ that only depend on $b_{1}, r_{1}$ and $r_{2}$ such that for any $i, j \leq p$, and $\gamma^{-1}=3 r_{1}^{-1}+r_{2}^{-1}$,

$$
\begin{aligned}
P\left(\left|\frac{1}{T} \sum_{t=1}^{T} u_{i t} u_{j t}-\sigma_{i j}\right| \geq s\right) \leq & T \exp \left(-\frac{(T s)^{\gamma}}{C_{1}}\right)+\exp \left(-\frac{T^{2} s^{2}}{C_{2}\left(1+T C_{3}\right)}\right) \\
& +\exp \left(-\frac{(T s)^{2}}{C_{4} T} \exp \left(\frac{(T s)^{\gamma(1-\gamma)}}{C_{5}(\log T s)^{\gamma}}\right)\right) .
\end{aligned}
$$


Using Bonferroni's method, we have

$$
\begin{aligned}
& P\left(\max _{i, j \leq p}\left|\frac{1}{T} \sum_{t=1}^{T} u_{i t} u_{j t}-\sigma_{i j}\right|>s\right) \\
& \quad \leq p^{2} \max _{i, j \leq p} P\left(\left|\frac{1}{T} \sum_{t=1}^{T} u_{i t} u_{j t}-\sigma_{i j}\right|>s\right) .
\end{aligned}
$$

Let $s=C \sqrt{(\log p) / T}$ for some $C>0$. It is not hard to check that when $(\log p)^{2 / \gamma-1}=o(T)$ (by assumption), for large enough $C$,

$$
p^{2} T \exp \left(-\frac{(T s)^{\gamma}}{C_{1}}\right)+p^{2} \exp \left(-\frac{(T s)^{2}}{C_{4} T} \exp \left(\frac{(T s)^{\gamma(1-\gamma)}}{C_{5}(\log T s)^{\gamma}}\right)\right)=o\left(\frac{1}{p^{2}}\right)
$$

and

$$
p^{2} \exp \left(-\frac{T^{2} s^{2}}{C_{2}\left(1+T C_{3}\right)}\right)=O\left(\frac{1}{p^{2}}\right)
$$

This proves (i).

(ii) For some $C_{1}^{\prime}>0$ such that

$$
P\left(\max _{i \leq p} \frac{1}{T} \sum_{t=1}^{T}\left(\widehat{u}_{i t}-u_{i t}\right)^{2}>C_{1}^{\prime} a_{T}^{2}\right)=O\left(\kappa_{1}(p, T)\right)
$$

under the event $\left\{\max _{i \leq p}\left|\frac{1}{T} \sum_{t=1}^{T} u_{i t}^{2}-\sigma_{i i}\right| \leq \max _{i \leq p} \sigma_{i i} / 4\right\} \cap\left\{\max _{i \leq p} \frac{1}{T} \times\right.$ $\left.\sum_{t=1}^{T}\left(\widehat{u}_{i t}-u_{i t}\right)^{2} \leq C_{1}^{\prime} a_{T}^{2}\right\}$, by the Cauchy-Schwarz inequality,

$$
\begin{aligned}
Z & \equiv \max _{i, j \leq p}\left|\frac{1}{T} \sum_{t=1}^{T}\left(\widehat{u}_{i t} \widehat{u}_{j t}-u_{i t} u_{j t}\right)\right| \\
& \leq \max _{i, j \leq p}\left|\frac{1}{T} \sum_{t=1}^{T}\left(\widehat{u}_{i t}-u_{i t}\right)\left(\widehat{u}_{j t}-u_{j t}\right)\right|+2 \max _{i, j \leq p}\left|\frac{1}{T} \sum_{t=1}^{T} u_{i t}\left(\widehat{u}_{j t}-u_{j t}\right)\right| \\
& \leq \max _{i \leq p} \frac{1}{T} \sum_{t=1}^{T}\left(\widehat{u}_{i t}-u_{i t}\right)^{2}+2 \sqrt{\max _{i \leq p} \frac{1}{T} \sum_{t=1}^{T} u_{i t}^{2}} \sqrt{\max _{i \leq p} \frac{1}{T} \sum_{t=1}^{T}\left(\widehat{u}_{i t}-u_{i t}\right)^{2}} \\
& \leq C_{1}^{\prime} a_{T}^{2}+2 \sqrt{\frac{5}{4} \max _{i \leq p} \sigma_{i i} \sqrt{C_{1}^{\prime} a_{T}^{2}} .}
\end{aligned}
$$

Since $a_{T}=o(1)$, when $C>3 \sqrt{C_{1}^{\prime} \max _{i \leq p} \sigma_{i i}}$, we have, for all large $T$,

$$
C a_{T}>C_{1}^{\prime} a_{T}^{2}+2 \sqrt{\frac{5}{4} \max _{i \leq p} \sigma_{i i}} \sqrt{C_{1}^{\prime} a_{T}^{2}}
$$


and

$$
\begin{aligned}
P\left(Z \leq C a_{T}\right) \geq & 1-P\left(\max _{i \leq p}\left|\frac{1}{T} \sum_{t=1}^{T} u_{i t}^{2}-\sigma_{i i}\right|>\max _{i \leq p} \sigma_{i i} / 4\right) \\
& -P\left(\max _{i \leq p} \frac{1}{T} \sum_{t=1}^{T}\left(\widehat{u}_{i t}-u_{i t}\right)^{2}>C_{1}^{\prime} a_{T}^{2}\right) .
\end{aligned}
$$

By part (i) and (A.2), $P\left(Z \leq C a_{T}\right) \geq 1-O\left(p^{-2}+\kappa_{1}(p, T)\right)$.

(iii) By (i) and (ii), there exists $C_{r}>0$, when $C>C_{r}$, the displayed inequalities in (i) and (ii) hold. Under the event $\left\{\max _{i, j \leq p}\left|\frac{1}{T} \sum_{t=1}^{T} u_{i t} u_{j t}-\sigma_{i j}\right| \leq\right.$ $C \sqrt{(\log p) / T}\} \cap\left\{\max _{i, j \leq p}\left|\frac{1}{T} \sum_{t=1}^{T} \widehat{u}_{i t} \widehat{u}_{j t}-u_{i t} u_{j t}\right| \leq C a_{T}\right\}$, by the triangular inequality,

$$
\begin{aligned}
\max _{i, j \leq p}\left|\widehat{\sigma}_{i j}-\sigma_{i j}\right| \leq \max _{i, j \leq p}\left|\frac{1}{T} \sum_{t=1}^{T} u_{i t} u_{j t}-\sigma_{i j}\right| \\
+\max _{i, j \leq p}\left|\frac{1}{T} \sum_{t=1}^{T} \widehat{u}_{i t} \widehat{u}_{j t}-u_{i t} u_{j t}\right| \\
\leq C\left(\sqrt{\frac{\log p}{T}}+a_{T}\right)
\end{aligned}
$$

Hence the desired result follows from part (i) and part (ii) of the lemma.

LEMMA A.4. Under Assumptions 2.1, 2.2,

$$
P\left(C_{L} \leq \min _{i j} \widehat{\theta}_{i j} \leq \max _{i j} \widehat{\theta}_{i j} \leq C_{U}\right) \geq 1-O\left(\frac{1}{p^{2}}+\kappa_{1}(p, T)+\kappa_{2}(p, T)\right),
$$

where

$$
\begin{aligned}
C_{L} & =\frac{1}{45} \min _{i j} \operatorname{var}\left(u_{i t} u_{j t}\right), \\
C_{U} & =3 \max _{i \leq p} \sigma_{i i}+4 \max _{i j} \operatorname{var}\left(u_{i t} u_{j t}\right) .
\end{aligned}
$$

PROOF. (i) Using Bernstein's inequality and the same argument as in the proof of Lemma A.3(i), there exists $C_{r}^{\prime}>0$, when $C>C_{r}^{\prime}$ and $(\log p)^{6 / \gamma-1}=o(T)$,

$$
P\left(\max _{i, j \leq p}\left|\frac{1}{T} \sum_{t=1}^{T}\left(u_{i t} u_{j t}-\sigma_{i j}\right)^{2}-\operatorname{var}\left(u_{i t} u_{j t}\right)\right|>C \sqrt{\frac{\log p}{T}}\right)=O\left(\frac{1}{p^{2}}\right) .
$$


For some $C>0$, under the event $\bigcap_{i=1}^{4} A_{i}$, where

$$
\begin{aligned}
& A_{1}=\left\{\max _{i, j \leq p}\left|\sigma_{i j}-\widehat{\sigma}_{i j}\right| \leq C\left(\sqrt{\frac{\log p}{T}}+a_{T}\right)\right\}, \\
& A_{2}=\left\{\max _{i \leq p, t \leq T}\left|\widehat{u}_{i t}-u_{i t}\right| \leq \min \left\{\frac{1}{2}, \sqrt{\left(20 \max _{i} \sigma_{i i}\right)^{-1} \min _{i j} \operatorname{var}\left(u_{i t} u_{j t}\right)}\right\}\right\}, \\
& A_{3}=\left\{\max _{i \leq p}\left|\frac{1}{T} \sum_{t=1}^{T} u_{i t}^{2}-\sigma_{i i}\right| \leq C \sqrt{\frac{\log p}{T}}\right\}, \\
& A_{4}=\left\{\max _{i, j \leq p}\left|\frac{1}{T} \sum_{t=1}^{T}\left(u_{i t} u_{j t}-\sigma_{i j}\right)^{2}-\operatorname{var}\left(u_{i t} u_{j t}\right)\right| \leq C \sqrt{\frac{\log p}{T}}\right\},
\end{aligned}
$$

we have, for any $i, j$, by adding and subtracting terms,

$$
\begin{aligned}
\widehat{\theta}_{i, j}= & \frac{1}{T} \sum_{t}\left(\widehat{u}_{i t} \widehat{u}_{j t}-\widehat{\sigma}_{i j}\right)^{2} \\
\leq & \frac{2}{T} \sum_{t}\left(\widehat{u}_{i t} \widehat{u}_{j t}-\sigma_{i j}\right)^{2}+2 \max _{i, j}\left(\sigma_{i j}-\widehat{\sigma}_{i j}\right)^{2} \\
\leq & \frac{4}{T} \sum_{t}\left(\widehat{u}_{i t}-u_{i t}\right)^{2} \widehat{u}_{j t}^{2}+\frac{4}{T} \sum_{t}\left(\widehat{u}_{j t}-u_{j t}\right)^{2} u_{i t}^{2} \\
& +\frac{4}{T} \sum_{t}\left(u_{i t} u_{j t}-\sigma_{i j}\right)^{2}+O\left(\frac{\log p}{T}+a_{T}^{2}\right) \\
\leq & 4 \max _{i t}\left|\widehat{u}_{i t}-u_{i t}\right|^{2}\left(\max _{i} \widehat{\sigma}_{i i}+\max _{i} \frac{1}{T} \sum_{t} u_{i t}^{2}\right) \\
& +4 \operatorname{var}\left(u_{i t} u_{j t}\right)+O\left(\sqrt{\frac{\log p}{T}}+\frac{\log p}{T}+a_{T}^{2}\right) \\
\leq & \left(2 C \sqrt{\frac{\log p}{T}}+C a_{T}+2 \max _{i} \sigma_{i i}\right) \\
& +4 \operatorname{var}\left(u_{i t} u_{j t}\right)+o(1),
\end{aligned}
$$

where the $O(\cdot)$ and $o(\cdot)$ terms are uniform in $p$ an $T$. Hence under $\bigcap_{i=1}^{4} A_{i}$, for all large enough $T, p$, uniformly in $i, j$, we have

$$
\widehat{\theta}_{i, j} \leq 3 \max _{i \leq p} \sigma_{i i}+4 \max _{i j} \operatorname{var}\left(u_{i t} u_{j t}\right) .
$$


Still by adding and subtracting terms, we obtain

$$
\begin{aligned}
& \frac{1}{T} \sum_{t}\left(u_{i t} u_{j t}-\sigma_{i j}\right)^{2} \\
& \quad \leq \frac{4}{T} \sum_{t}\left(u_{i t} u_{j t}-\widehat{u}_{i t} \widehat{u}_{j t}\right)^{2}+\frac{4}{T} \sum_{t}\left(\widehat{u}_{i t} \widehat{u}_{j t}-\widehat{\sigma}_{i j}\right)^{2}+4\left(\sigma_{i j}-\widehat{\sigma}_{i j}\right)^{2} \\
& \quad \leq \frac{8}{T} \sum_{t} u_{i t}^{2}\left(u_{j t}-\widehat{u}_{j t}\right)^{2}+\frac{8}{T} \sum_{t} \widehat{u}_{j t}^{2}\left(u_{i t}-\widehat{u}_{i t}\right)^{2}+4 \widehat{\theta}_{i j}+O\left(\frac{\log p}{T}+a_{T}^{2}\right) \\
& \quad \leq 8 \max _{i t}\left|\widehat{u}_{i t}-u_{i t}\right|^{2}\left(\max _{i} \widehat{\sigma}_{i i}+\max _{j} \frac{1}{T} \sum_{t} u_{j t}^{2}\right)+4 \widehat{\theta}_{i j}+o(1) .
\end{aligned}
$$

Under the event $\bigcap_{i=1}^{4} A_{i}$, we have

$$
\begin{aligned}
4 \widehat{\theta}_{i j}+o(1) \geq & \min _{i j} \operatorname{var}\left(u_{i t} u_{j t}\right)-C \sqrt{\frac{\log p}{T}} \\
& -8 \max _{i t}\left|\widehat{u}_{i t}-u_{i t}\right|^{2}\left[2 C \sqrt{\frac{\log p}{T}}+C a_{T}+2 \max _{i} \sigma_{i i}\right] \\
\geq & \frac{1}{10} \min _{i j} \operatorname{var}\left(u_{i t} u_{j t}\right) .
\end{aligned}
$$

Hence for all large $T, p$, uniformly in $i, j$, we have $\widehat{\theta}_{i j} \geq \frac{1}{45} \min _{i j} \operatorname{var}\left(u_{i t} u_{j t}\right)$.

Finally, by Lemma A.3 and Assumption 2.2,

$$
P\left(\bigcap_{i=1}^{4} A_{i}\right) \geq 1-O\left(\frac{1}{p^{2}}+\kappa_{1}(p, T)+\kappa_{2}(p, T)\right),
$$

which completes the proof.

A.2. Proof of Theorem 2.1. (i) For the operator norm, we have

$$
\left\|\widehat{\boldsymbol{\Sigma}}_{u}^{\mathcal{T}}-\boldsymbol{\Sigma}_{u}\right\| \leq \max _{i \leq p} \sum_{j=1}^{p}\left|\widehat{\sigma}_{i j} I\left(\left|\widehat{\sigma}_{i j}\right| \geq \omega_{T} \widehat{\theta}_{i j}^{1 / 2}\right)-\sigma_{i j}\right| .
$$

By Lemma A.3(iii), there exists $C_{1}>0$ such that the event

$$
A_{1}^{\prime}=\left\{\max _{i, j \leq p}\left|\widehat{\sigma}_{i j}-\sigma_{i j}\right| \leq C_{1}\left(\sqrt{\frac{\log p}{T}}+a_{T}\right)\right\}
$$

occurs with probability $P\left(A_{1}^{\prime}\right) \geq 1-O\left(\frac{1}{p^{2}}+\kappa_{1}(p, T)\right)$. Let $C>0$ be such that $C \sqrt{C_{L}}>2 C_{1}$, where $C_{L}$ is defined in Lemma A.4. Let $\omega_{T}=C\left(\sqrt{\frac{\log p}{T}}+a_{T}\right)$, 


$$
\begin{aligned}
b_{T}=C_{1}\left(\sqrt{\frac{\log p}{T}}+a_{T}\right), \text { then } \sqrt{C_{L}} \omega_{T}>2 b_{T}, \text { and by Lemma A.4, } \\
P\left(\min _{i j} \widehat{\theta}_{i j}^{1 / 2} \omega_{T}>2 b_{T}\right) \geq P\left(\min _{i j} \widehat{\theta}_{i j}^{1 / 2}>\sqrt{C_{L}}\right) \\
\geq 1-O\left(\frac{1}{p}+\kappa_{1}(p, T)+\kappa_{2}(p, T)\right) .
\end{aligned}
$$

Define the following events:

$$
\begin{aligned}
& A_{2}^{\prime}=\left\{\min _{i j} \widehat{\theta}_{i j}^{1 / 2} \omega_{T}>2 b_{T}\right\}, \\
& A_{3}^{\prime}=\left\{\max _{i j} \widehat{\theta}_{i j}^{1 / 2} \leq C_{U}^{1 / 2}\right\},
\end{aligned}
$$

where $C_{U}$ is defined in Lemma A.4. Under $\bigcap_{i=1}^{3} A_{i}^{\prime}$, the event $\left|\widehat{\sigma}_{i j}\right| \geq \omega_{T} \widehat{\theta}_{i j}^{1 / 2}$ implies $\left|\sigma_{i j}\right| \geq b_{T}$, and the event $\left|\widehat{\sigma}_{i j}\right|<\omega_{T} \widehat{\theta}_{i j}^{1 / 2}$ implies $\left|\sigma_{i j}\right|<b_{T}+\sqrt{C_{U}} \omega_{T}$. We thus have, uniformly in $i \leq p$, under $\bigcap_{i=1}^{3} A_{i}^{\prime}$,

$$
\begin{aligned}
\left\|\widehat{\boldsymbol{\Sigma}}_{u}^{\mathcal{T}}-\boldsymbol{\Sigma}_{u}\right\| & \leq \sum_{j=1}^{p}\left|\widehat{\sigma}_{i j} I\left(\left|\widehat{\sigma}_{i j}\right| \geq \omega_{T} \widehat{\theta}_{i j}^{1 / 2}\right)-\sigma_{i j}\right| \\
& \leq \sum_{j=1}^{p}\left|\widehat{\sigma}_{i j}-\sigma_{i j}\right| I\left(\left|\widehat{\sigma}_{i j}\right| \geq \omega_{T} \widehat{\theta}_{i j}^{1 / 2}\right)+\sum_{j=1}^{p}\left|\sigma_{i j}\right| I\left(\left|\widehat{\sigma}_{i j}\right|<\omega_{T} \widehat{\theta}_{i j}^{1 / 2}\right) \\
& \leq \sum_{j=1}^{p}\left|\widehat{\sigma}_{i j}-\sigma_{i j}\right| I\left(\left|\sigma_{i j}\right| \geq b_{T}\right)+\sum_{j=1}^{p}\left|\sigma_{i j}\right| I\left(\left|\sigma_{i j}\right|<b_{T}+\sqrt{C_{U}} \omega_{T}\right) \\
& \leq b_{T} m_{T}+\left(b_{T}+\sqrt{C_{U}} \omega_{T}\right) m_{T} \\
& \leq\left(\sqrt{C_{L}}+\sqrt{C_{U}}\right) \omega_{T} m_{T} .
\end{aligned}
$$

By Lemmas A.3(iii) and A.4, $P\left(\bigcap_{i=1}^{3} A_{i}^{\prime}\right) \geq 1-O\left(\frac{1}{p^{2}}+\kappa_{1}(p, T)+\kappa_{2}(p, T)\right)$, which proves the result.

(ii) By part (i) of the theorem, there exists some $C>0$,

$$
P\left(\left\|\widehat{\boldsymbol{\Sigma}}_{u}^{\mathcal{T}}-\boldsymbol{\Sigma}_{u}\right\|>C \omega_{T} m_{T}\right)=O\left(\frac{1}{p^{2}}+\kappa_{1}(p, T)+\kappa_{2}(p, T)\right) .
$$

By Lemma A.1,

$$
\begin{aligned}
P\left(\lambda_{\min }\left(\widehat{\boldsymbol{\Sigma}}_{u}^{\mathcal{T}}\right) \geq 0.5 \lambda_{\min }\left(\boldsymbol{\Sigma}_{u}\right)\right) & \geq P\left(\left\|\widehat{\boldsymbol{\Sigma}}_{u}^{\mathcal{T}}-\boldsymbol{\Sigma}_{u}\right\| \leq 0.5 \lambda_{\min }\left(\boldsymbol{\Sigma}_{u}\right)\right) \\
& \geq 1-O\left(\frac{1}{p^{2}}+\kappa_{1}(p, T)+\kappa_{2}(p, T)\right) .
\end{aligned}
$$


In addition, when $\omega_{T} m_{T}=o(1)$,

$$
\begin{aligned}
& P\left(\left\|\left(\widehat{\boldsymbol{\Sigma}}_{u}^{\mathcal{T}}\right)^{-1}-\boldsymbol{\Sigma}_{u}^{-1}\right\| \leq 2\left\|\boldsymbol{\Sigma}_{u}^{-1}\right\| C \omega_{T} m_{T}\right) \\
& \geq P\left(\left\|\left(\widehat{\boldsymbol{\Sigma}}_{u}^{\mathcal{T}}\right)^{-1}-\boldsymbol{\Sigma}_{u}^{-1}\right\| \leq 2\left\|\boldsymbol{\Sigma}_{u}^{-1}\right\| \cdot\left\|\widehat{\boldsymbol{\Sigma}}_{u}^{\mathcal{T}}-\boldsymbol{\Sigma}_{u}\right\|,\left\|\widehat{\boldsymbol{\Sigma}}_{u}^{\mathcal{T}}-\boldsymbol{\Sigma}_{u}\right\| \leq C \omega_{T} m_{T}\right) \\
& \geq P\left(\left\|\left(\widehat{\boldsymbol{\Sigma}}_{u}^{\mathcal{T}}\right)^{-1}-\boldsymbol{\Sigma}_{u}^{-1}\right\| \leq 2\left\|\boldsymbol{\Sigma}_{u}^{-1}\right\| \cdot\left\|\widehat{\boldsymbol{\Sigma}}_{u}^{\mathcal{T}}-\boldsymbol{\Sigma}_{u}\right\|\right) \\
& \quad-P\left(\left\|\widehat{\boldsymbol{\Sigma}}_{u}^{\mathcal{T}}-\boldsymbol{\Sigma}_{u}\right\|>C \omega_{T} m_{T}\right) \\
& \geq P\left(\left\|\widehat{\boldsymbol{\Sigma}}_{u}^{\mathcal{T}}-\boldsymbol{\Sigma}_{u}\right\| \leq 0.5 \lambda_{\min }\left(\boldsymbol{\Sigma}_{u}\right)\right) \\
& \quad-O\left(\frac{1}{p^{2}}+\kappa_{1}(p, T)+\kappa_{2}(p, T)\right) \\
& \\
& \geq-O\left(\frac{1}{p^{2}}+\kappa_{1}(p, T)+\kappa_{2}(p, T)\right),
\end{aligned}
$$

where the third inequality follows from Lemma A.1 as well.

\section{APPENDIX B: PROOFS FOR SECTION 3}

\section{B.1. Proof of Theorem 3.1.}

LEMmA B.1. There exists $C_{1}>0$ such that:

(i)

$$
P\left(\max _{i, j \leq K}\left|\frac{1}{T} \sum_{t=1}^{T} f_{i t} f_{j t}-E f_{i t} f_{j t}\right|>C_{1} \sqrt{\frac{\log T}{T}}\right)=O\left(\frac{1}{T^{2}}\right),
$$

(ii)

$$
P\left(\max _{k \leq K, i \leq p}\left|\frac{1}{T} \sum_{t=1}^{T} f_{k t} u_{i t}\right|>C_{1} \sqrt{\frac{\log p}{T}}\right)=O\left(\frac{1}{p^{2}}\right) .
$$

ProOF. (i) Let $Z_{i j}=\frac{1}{T} \sum_{t=1}^{T}\left(f_{i t} f_{j t}-E f_{i t} f_{j t}\right)$. We bound $\max _{i j}\left|Z_{i j}\right|$ using a Bernstein-type inequality. Lemma A.2 implies that for any $i$ and $j \leq K$, $f_{i t} f_{j t}$ satisfies the exponential tail condition (3.3) with parameter $r_{3} / 3$. Let $r_{4}^{-1}=$ $3 r_{3}^{-1}+r_{2}^{-1}$, where $r_{2}>0$ is the parameter in the strong mixing condition. By Assumption 3.3, $r_{4}<1$, and by the Bernstein inequality for weakly dependent data in Merlevède, Peligrad and Rio [(2009), Theorem 1], there exist $C_{i}>0, i=1, \ldots, 5$, for any $s>0$

$$
\begin{aligned}
\max _{i, j} P\left(\left|Z_{i j}\right|>s\right) \leq & T \exp \left(-\frac{(T s)^{r_{4}}}{C_{1}}\right)+\exp \left(-\frac{T^{2} s^{2}}{C_{2}\left(1+T C_{3}\right)}\right) \\
& +\exp \left(-\frac{(T s)^{2}}{C_{4} T} \exp \left(\frac{(T s)^{r_{4}\left(1-r_{4}\right)}}{C_{5}(\log T s)^{r_{4}}}\right)\right) .
\end{aligned}
$$


Using the Bonferroni inequality,

$$
P\left(\max _{i \leq K, j \leq K}\left|Z_{i j}\right|>s\right) \leq K^{2} \max _{i, j} P\left(\left|Z_{i j}\right|>s\right) .
$$

Let $s=C \sqrt{(\log T) / T}$. For all large enough $C$, since $K^{2}=o(T)$,

$$
\begin{aligned}
T K^{2} \exp \left(-\frac{(T s)^{r_{4}}}{C_{1}}\right)+K^{2} \exp \left(-\frac{(T s)^{2}}{C_{4} T} \exp \left(\frac{(T s)^{r_{4}\left(1-r_{4}\right)}}{C_{5}(\log T s)^{r_{4}}}\right)\right) & =o\left(\frac{1}{T^{2}}\right), \\
K^{2} \exp \left(-\frac{T^{2} s^{2}}{C_{2}\left(1+T C_{3}\right)}\right) & =O\left(\frac{1}{T^{2}}\right) .
\end{aligned}
$$

This proves part (i).

(ii) By Lemma A.2, and Assumptions 2.1(iii) and 3.3(ii), $Z_{k i, t}^{\prime} \equiv f_{k t} u_{i t}$ satisfies the exponential tail condition (2.6) for the tail parameter $2 r_{1} r_{3} /\left(3 r_{1}+3 r_{3}\right)$, as well as the strong mixing condition with parameter $r_{2}$. Hence again we can apply the Bernstein inequality for weakly dependent data in Merlevède, Peligrad and Rio [(2009), Theorem 1] and the Bonferroni method on $Z_{k i, t}^{\prime}$ similar to (B.1) with the parameter $\gamma_{2}^{-1}=1.5 r_{1}^{-1}+1.5 r_{3}^{-1}+r_{2}^{-1}$. It follows from $3 r_{1}^{-1}+r_{2}^{-1}>1$ and $3 r_{3}^{-1}+r_{2}^{-1}>1$ that $\gamma_{2}<1$. Thus when $s=C \sqrt{(\log p) / T}$ for large enough $C$, the term

$$
p K \exp \left(-\frac{T^{2} s^{2}}{C_{2}\left(1+T C_{3}\right)}\right) \leq p^{-2},
$$

and the rest terms on the right-hand side of the inequality, multiplied by $p K$ are of order $o\left(p^{-2}\right)$. Hence when $(\log p)^{2 / \gamma_{2}-1}=o(T)$ (which is implied by the theorem's assumption) and $K=o(p)$, there exists $C^{\prime}>0$,

$$
P\left(\max _{k \leq K, i \leq p}\left|\frac{1}{T} \sum_{t=1}^{T} f_{k t} u_{i t}\right|>C^{\prime} \sqrt{\frac{\log p}{T}}\right)=O\left(\frac{1}{p^{2}}\right) .
$$

Proof of Lemma 3.1. (i) Since $K \sqrt{\log T}=o(\sqrt{T})$, and $\lambda_{\min }\left(E \mathbf{f}_{t} \mathbf{f}_{t}^{\prime}\right)$ is bounded away from zero, for large enough $T$, by Lemma B.1(i),

$$
\begin{aligned}
& P\left(\left\|\frac{1}{T} \mathbf{X} \mathbf{X}^{\prime}-E \mathbf{f}_{t} \mathbf{f}_{t}^{\prime}\right\| \leq 0.5 \lambda_{\min }\left(E \mathbf{f}_{t} \mathbf{f}_{t}^{\prime}\right)\right) \\
& \quad \geq P\left(K \max _{i \leq K, j \leq K}\left|\frac{1}{T} \sum_{t=1}^{T} f_{i t} f_{j t}-E f_{i t} f_{j t}\right| \leq 0.5 \lambda_{\min }\left(E \mathbf{f}_{t} \mathbf{f}_{t}^{\prime}\right)\right) \\
& \quad \geq 1-O\left(\frac{1}{T^{2}}\right) .
\end{aligned}
$$

Hence by Lemma A.1,

$$
P\left(\lambda_{\min }\left(T^{-1} \mathbf{X} \mathbf{X}^{\prime}\right) \geq 0.5 \lambda_{\min }\left(E \mathbf{f}_{t} \mathbf{f}_{t}^{\prime}\right)\right) \geq 1-O\left(\frac{1}{T^{2}}\right) .
$$


As $\widehat{\mathbf{b}}_{i}-\mathbf{b}_{i}=\left(\mathbf{X} \mathbf{X}^{\prime}\right)^{-1} \mathbf{X} \mathbf{u}_{i}$, we have $\left\|\widehat{\mathbf{b}}_{i}-\mathbf{b}_{i}\right\|^{2}=\mathbf{u}_{i}^{\prime} \mathbf{X}^{\prime}\left(\mathbf{X} \mathbf{X}^{\prime}\right)^{-2} \mathbf{X} \mathbf{u}_{i}$. For $C^{\prime}>0$ such that (B.2) holds, under the event

$$
A \equiv\left\{\max _{k \leq K, i \leq p}\left|\frac{1}{T} \sum_{t=1}^{T} f_{k t} u_{i t}\right| \leq C^{\prime} \sqrt{\frac{\log p}{T}}\right\} \cap\left\{\lambda_{\min }\left(T^{-1} \mathbf{X X}^{\prime}\right) \geq 0.5 \lambda_{\min }\left(E \mathbf{f}_{t} \mathbf{f}_{t}^{\prime}\right)\right\},
$$

we have

$$
\begin{aligned}
\left\|\widehat{\mathbf{b}}_{i}-\mathbf{b}_{i}\right\|^{2} & \leq \frac{4}{\lambda_{\min }\left(E \mathbf{f}_{t} \mathbf{f}_{t}^{\prime}\right)^{2}} \sum_{k=1}^{K}\left(\frac{1}{T} \sum_{t=1}^{T} f_{k t} u_{i t}\right)^{2} \\
& \leq \frac{4 K}{\lambda_{\min }\left(E \mathbf{f}_{t} \mathbf{f}_{t}^{\prime}\right)^{2}} \max _{k \leq K, i \leq p}\left(\frac{1}{T} \sum_{t=1}^{T} f_{k t} u_{i t}\right)^{2} \\
& \leq \frac{4 K C^{\prime 2} \log p}{\lambda_{\min }\left(E \mathbf{f}_{t} \mathbf{f}_{t}^{\prime}\right)^{2} T} .
\end{aligned}
$$

The desired result then follows from that $P(A) \geq 1-O\left(\frac{1}{T^{2}}+\frac{1}{p^{2}}\right)$.

(ii) For $C>\max _{i \leq K} E f_{i t}^{2}$, we have, by Lemma B.1(i),

$$
\begin{aligned}
P\left(\frac{1}{T} \sum_{t}\left\|\mathbf{f}_{t}\right\|^{2}>C K\right) & \leq P\left(K \max _{k \leq K}\left|\frac{1}{T} \sum_{t=1}^{T} f_{k t}^{2}-E f_{k t}^{2}\right|+K \max _{k \leq K} E f_{k t}^{2}>C K\right) \\
& =O\left(\frac{1}{T^{2}}\right) .
\end{aligned}
$$

The result then follows from

$$
\max _{i \leq p} \frac{1}{T} \sum_{t=1}^{T}\left|u_{i t}-\widehat{u}_{i t}\right|^{2} \leq \max _{i \leq p} \frac{1}{T} \sum_{t}\left\|\mathbf{f}_{t}\right\|^{2}\left\|\widehat{\mathbf{b}}_{i}-\mathbf{b}_{i}\right\|^{2}
$$

and part (i).

(iii) By Assumption 3.3, for any $s>0$,

$$
\begin{aligned}
P\left(\max _{t \leq T}\left\|\mathbf{f}_{t}\right\|>s\right) & \leq T P\left(\left\|\mathbf{f}_{t}\right\|>s\right) \leq T K \max _{k \leq K} P\left(f_{k t}^{2}>s^{2} / K\right) \\
& \leq T K \exp \left(-\left(\frac{s}{b_{2} \sqrt{K}}\right)^{r_{3}}\right) .
\end{aligned}
$$

When $s \geq C \sqrt{K}(\log T)^{1 / r_{3}}$ for large enough $C$, that is, $C^{r_{3}}>4 b_{2}^{r_{3}}$,

$$
P\left(\max _{t \leq T}\left\|\mathbf{f}_{t}\right\|>C \sqrt{K}(\log T)^{1 / r_{3}}\right) \leq T^{-2} .
$$

The result then follows from

$$
\max _{t \leq T, i \leq p}\left|u_{i t}-\widehat{u}_{i t}\right|=\max _{t \leq T, i \leq p}\left|\left(\widehat{\mathbf{b}}_{i}-\mathbf{b}_{i}\right)^{\prime} \mathbf{f}_{t}\right| \leq \max _{i}\left\|\widehat{\mathbf{b}}_{i}-\mathbf{b}_{i}\right\| \max _{t}\left\|\mathbf{f}_{t}\right\|
$$


and Lemma 3.1(i).

Proof of TheOrem 3.1. Theorem 3.1 follows immediately from Theorem 2.1 and Lemma 3.1.

B.2. Proof of Theorem 3.2, part (i). Define

$$
\begin{aligned}
\mathbf{D}_{T} & =\widehat{\operatorname{cov}}\left(\mathbf{f}_{t}\right)-\operatorname{cov}\left(\mathbf{f}_{t}\right), \quad \mathbf{C}_{T}=\widehat{\mathbf{B}}-\mathbf{B}, \\
\mathbf{E} & =\left(\mathbf{u}_{1}, \ldots, \mathbf{u}_{T}\right) .
\end{aligned}
$$

We have

$$
\begin{aligned}
\left\|\widehat{\Sigma}^{\mathcal{T}}-\Sigma\right\|_{\Sigma}^{2} \leq & 4\left\|\mathbf{B} \mathbf{D}_{T} \mathbf{B}^{\prime}\right\|_{\Sigma}^{2}+24\left\|\mathbf{B} \widehat{\operatorname{cov}}(\mathbf{f}) \mathbf{C}_{T}^{\prime}\right\|_{\Sigma}^{2} \\
& +16\left\|\mathbf{C}_{T} \widehat{\operatorname{cov}}(\mathbf{f}) \mathbf{C}_{T}^{\prime}\right\|_{\Sigma}^{2}+2\left\|\widehat{\Sigma}_{u}^{\mathcal{T}}-\boldsymbol{\Sigma}_{u}\right\|_{\Sigma}^{2}
\end{aligned}
$$

We bound the terms on the right-hand side in the following lemmas.

LEMMA B.2. There exists $C>0$, such that:

(i)

$$
P\left(\left\|\mathbf{D}_{T}\right\|_{F}^{2}>\frac{C K^{2} \log T}{T}\right)=O\left(T^{-2}\right)
$$

(ii)

$$
P\left(\left\|\mathbf{C}_{T}\right\|_{F}^{2}>\frac{C K p \log p}{T}\right)=O\left(T^{-2}+p^{-2}\right)
$$

PROOF. (i) Similarly to the proof of Lemma B.1(i), it can be shown that there exists $C_{1}>0$,

$$
P\left(\max _{i \leq K}\left|\frac{1}{T} \sum_{t=1}^{T} f_{i t}-E f_{i t}\right|>C_{1} \sqrt{\frac{\log T}{T}}\right)=O\left(T^{-2}\right) .
$$

Hence $\sup _{K} \max _{i \leq K} E\left|f_{i t}\right|<\infty$ implies that there exists $C>0$ such that

$$
P\left(\max _{i, j \leq K}\left|\frac{1}{T} \sum_{t=1}^{T} f_{i t} \frac{1}{T} \sum_{t=1}^{T} f_{j t}-E f_{i t} E f_{j t}\right|>C \sqrt{\frac{\log T}{T}}\right)=O\left(T^{-2}\right) .
$$

The result then follows from Lemma B.1(i) and that

$$
\begin{aligned}
\left\|\mathbf{D}_{T}\right\|_{F}^{2} \leq K^{2}\left(\max _{i, j \leq K}\left|\frac{1}{T} \sum_{t=1}^{T} f_{i t} f_{j t}-E f_{i t} f_{j t}\right|^{2}\right. \\
\left.\quad+\max _{i, j \leq K}\left|\frac{1}{T} \sum_{t=1}^{T} f_{i t} \frac{1}{T} \sum_{t=1}^{T} f_{j t}-E f_{i t} E f_{j t}\right|^{2}\right) .
\end{aligned}
$$


(ii) We have $\mathbf{C}_{T}=\mathbf{E X}^{\prime}\left(\mathbf{X X}^{\prime}\right)^{-1}$. By Lemma B.1(ii), there exists $C^{\prime}>0$ such that

$$
P\left(\max _{k, i}\left|\frac{1}{T} \sum_{t=1}^{T} f_{k t} u_{i t}\right|>C^{\prime} \sqrt{\frac{\log p}{T}}\right)=O\left(p^{-2}\right) .
$$

Under the event

$$
A=\left\{\max _{k, i}\left|\frac{1}{T} \sum_{t=1}^{T} f_{k t} u_{i t}\right| \leq C^{\prime} \sqrt{\frac{\log p}{T}}\right\} \cap\left\{\lambda_{\min }\left(T^{-1} \mathbf{X} \mathbf{X}^{\prime}\right) \geq 0.5 \lambda_{\min }\left(E \mathbf{f}_{t} \mathbf{f}_{t}^{\prime}\right)\right\},
$$

$\left\|\mathbf{C}_{T}\right\|_{F}^{2} \leq 4 \lambda_{\min }^{-2}\left(E \mathbf{f}_{t} \mathbf{f}_{t}^{\prime}\right) C^{\prime 2} p K(\log p) / T$, which proves the result since $\lambda_{\min }\left(E \mathbf{f}_{t} \mathbf{f}_{t}^{\prime}\right)$ is bounded away from zero and $P(A) \geq 1-O\left(T^{-2}+p^{-2}\right)$ due to (B.4).

Lemma B.3. There exists $C>0$ such that:

(i)

$$
\begin{aligned}
& P\left(\left\|\mathbf{B D}_{T} \mathbf{B}^{\prime}\right\|_{\Sigma}^{2}+\left\|\mathbf{B} \widehat{\operatorname{cov}}\left(\mathbf{f}_{t}\right) \mathbf{C}_{T}^{\prime}\right\|_{\Sigma}^{2}>\frac{C K \log p}{T}+\frac{C K^{2} \log T}{T p}\right) \\
& \quad=O\left(T^{-2}+p^{-2}\right) ;
\end{aligned}
$$

(ii)

$$
P\left(\left\|\mathbf{C}_{T} \widehat{\operatorname{cov}}(\mathbf{f}) \mathbf{C}_{T}^{\prime}\right\|_{\Sigma}^{2}>\frac{C p K^{2}(\log p)^{2}}{T^{2}}\right)=O\left(T^{-2}+p^{-2}\right) .
$$

PROOF. (i) The same argument in Fan, Fan and Lv [(2008), proof of Theorem 2] implies that

$$
\left\|\mathbf{B}^{\prime} \boldsymbol{\Sigma}^{-1} \mathbf{B}\right\| \leq 2\left\|\operatorname{cov}\left(\mathbf{f}_{t}\right)^{-1}\right\|=O(1) .
$$

Hence

$$
\begin{aligned}
\left\|\mathbf{B D}_{T} \mathbf{B}^{\prime}\right\|_{\Sigma}^{2} & =p^{-1} \operatorname{tr}\left(\boldsymbol{\Sigma}^{-1 / 2} \mathbf{B} \mathbf{D}_{T} \mathbf{B}^{\prime} \boldsymbol{\Sigma}^{-1} \mathbf{B D}_{T} \mathbf{B}^{\prime} \boldsymbol{\Sigma}^{-1 / 2}\right) \\
& =p^{-1} \operatorname{tr}\left(\mathbf{D}_{T} \mathbf{B}^{\prime} \boldsymbol{\Sigma}^{-1} \mathbf{B D}_{T} \mathbf{B}^{\prime} \boldsymbol{\Sigma}^{-1} \mathbf{B}\right) \\
& \leq p^{-1}\left\|\mathbf{D}_{T} \mathbf{B}^{\prime} \boldsymbol{\Sigma}^{-1} \mathbf{B}\right\|_{F}^{2} \\
& \leq O\left(p^{-1}\right)\left\|\mathbf{D}_{T}\right\|_{F}^{2} .
\end{aligned}
$$

On the other hand,

(B.7) $\left\|\mathbf{B} \widehat{\operatorname{cov}}(\mathbf{f}) \mathbf{C}_{T}^{\prime}\right\|_{\Sigma}^{2} \leq 8 T^{-2}\left\|\mathbf{B X X} \mathbf{X}^{\prime} \mathbf{C}_{T}^{\prime}\right\|_{\Sigma}^{2}+8 T^{-4}\left\|\mathbf{B X 1 1}^{\prime} \mathbf{X}^{\prime} \mathbf{C}_{T}^{\prime}\right\|_{\Sigma}^{2}$.

Respectively,

$$
\begin{gathered}
\left\|\mathbf{B X X}^{\prime} \mathbf{C}_{T}^{\prime}\right\|_{\Sigma}^{2} \leq p^{-1}\left\|\mathbf{X X} \mathbf{X}_{T}^{\prime} \mathbf{C}^{-1}\right\|_{F}\left\|\mathbf{C}_{T} \mathbf{X X}^{\prime} \mathbf{B}^{\prime} \boldsymbol{\Sigma}^{-1} \mathbf{B}\right\|_{F} \\
\left\|\mathbf{B X X 1 1}^{\prime} \mathbf{X}^{\prime} \mathbf{C}_{T}^{\prime}\right\|_{\Sigma}^{2} \leq p^{-1}\left\|\mathbf{X 1 1}^{\prime} \mathbf{X}^{\prime} \mathbf{C}_{T}^{\prime} \boldsymbol{\Sigma}^{-1}\right\|_{F}\left\|\mathbf{C}_{T} \mathbf{X 1 1}^{\prime} \mathbf{X}^{\prime} \mathbf{B}^{\prime} \boldsymbol{\Sigma}^{-1} \mathbf{B}\right\|_{F}
\end{gathered}
$$


By Lemma B.1(i), and $E \mathbf{f}_{t} \mathbf{f}_{t}^{\prime}<\infty, P\left(\left\|\mathbf{X} \mathbf{X}^{\prime}\right\|>T C\right)=O\left(T^{-2}\right)$ for some $C>0$. Hence, Lemma B.2(ii) implies

$$
P\left(\left\|\mathbf{B X X} \mathbf{X}^{\prime} \mathbf{C}^{\prime}\right\|_{\Sigma}^{2}>C^{\prime} T K \log p\right)=O\left(T^{-2}+p^{-2}\right)
$$

for some $C^{\prime}>0$. In addition, the eigenvalues of $\widehat{\operatorname{cov}}\left(\mathbf{f}_{t}\right)=T^{-1} \mathbf{X} \mathbf{X}^{\prime}-T^{-2} \mathbf{X 1 1}^{\prime} \mathbf{X}^{\prime}$ are all bounded away from both zero and infinity with probability at least $1-$ $O\left(T^{-2}\right)$ [implied by Lemmas B.1(i), A.1 and Assumption 3.4]. Hence for some $C_{1}>0$, with probability ast least $1-O\left(T^{-2}\right)$,

$$
\left\|\mathbf{X} 11^{\prime} \mathbf{X}^{\prime}\right\| \leq\left\|T \mathbf{X} \mathbf{X}^{\prime}\right\| \leq T^{2} C_{1},
$$

$$
\left\|\mathbf{B X 1 1} \mathbf{X}^{\prime} \mathbf{C}_{T}^{\prime}\right\|_{\Sigma}^{2} \leq O\left(p^{-1}\right)\left\|\mathbf{X 1 1}^{\prime} \mathbf{X}^{\prime}\right\|^{2}\left\|\mathbf{C}_{T}\right\|_{F}^{2} .
$$

The result then follows from the combination of (B.6)-(B.10) and Lemma B.2.

(ii) Straightforward calculation yields

$$
\begin{aligned}
p\left\|\mathbf{C}_{T} \widehat{\operatorname{cov}}(\mathbf{f}) \mathbf{C}_{T}^{\prime}\right\|_{\Sigma}^{2} & =\operatorname{tr}\left(\mathbf{C}_{T} \widehat{\operatorname{cov}}(\mathbf{f}) \mathbf{C}_{T}{ }^{\prime} \boldsymbol{\Sigma}^{-1} \mathbf{C}_{T} \widehat{\operatorname{cov}}(\mathbf{f}) \mathbf{C}_{T}{ }^{\prime} \boldsymbol{\Sigma}^{-1}\right) \\
& \leq\left\|\mathbf{C}_{T} \widehat{\operatorname{cov}}(\mathbf{f}) \mathbf{C}_{T}{ }^{\prime} \boldsymbol{\Sigma}^{-1}\right\|_{F}^{2} \\
& \leq \lambda_{\max }^{2}\left(\boldsymbol{\Sigma}^{-1}\right) \lambda_{\max }^{2}\left(\widehat{\operatorname{cov}}\left(\mathbf{f}_{t}\right)\right)\left\|\mathbf{C}_{T}\right\|_{F}^{4} .
\end{aligned}
$$

Since $\left\|\operatorname{cov}\left(\mathbf{f}_{t}\right)\right\|$ is bounded, by Lemma B.1(i), $\lambda_{\max }^{2}\left(\widehat{\operatorname{cov}}\left(\mathbf{f}_{t}\right)\right)$ is bounded with probability at least $1-O\left(T^{-2}\right)$. The result again follows from Lemma B.2(ii).

Proof of TheOREM 3.2, PART (i). (a) We have

$$
\begin{aligned}
\left\|\widehat{\boldsymbol{\Sigma}}_{u}^{\mathcal{T}}-\boldsymbol{\Sigma}_{u}\right\|_{\Sigma} & =p^{-1 / 2}\left\|\boldsymbol{\Sigma}^{-1 / 2}\left(\widehat{\boldsymbol{\Sigma}}_{u}^{\mathcal{T}}-\boldsymbol{\Sigma}_{u}\right) \boldsymbol{\Sigma}^{-1 / 2}\right\|_{F} \\
& \leq\left\|\boldsymbol{\Sigma}^{-1 / 2}\left(\widehat{\boldsymbol{\Sigma}}_{u}^{\mathcal{T}}-\boldsymbol{\Sigma}_{u}\right) \boldsymbol{\Sigma}^{-1 / 2}\right\| \\
& \leq\left\|\widehat{\boldsymbol{\Sigma}}_{u}^{\mathcal{T}}-\boldsymbol{\Sigma}_{u}\right\| \cdot \lambda_{\max }\left(\boldsymbol{\Sigma}^{-1}\right) .
\end{aligned}
$$

Therefore, (B.5), (B.11), Theorem 3.1 and Lemmas B.2, B.3 yield the result, with the fact that [assuming $\log T=o(p)$ ]

$$
\begin{aligned}
& \frac{K \log p}{T}+\frac{K^{2} \log T}{T p}+\frac{p K^{2}(\log p)^{2}}{T^{2}}+\frac{m_{T}^{2} K^{2} \log p}{T} \\
& =O\left(\frac{p K^{2}(\log p)^{2}}{T^{2}}+\frac{m_{T}^{2} K^{2} \log p}{T}\right) .
\end{aligned}
$$

(b) For the infinity norm, it is straightforward to find that

$$
\begin{aligned}
\left\|\widehat{\boldsymbol{\Sigma}}^{\mathcal{T}}-\boldsymbol{\Sigma}\right\|_{\mathrm{MAX}} \leq & \left\|2 \mathbf{C}_{T} \operatorname{cov}\left(\mathbf{f}_{t}\right) \mathbf{B}^{\prime}\right\|_{\mathrm{MAX}}+\left\|\mathbf{B} \mathbf{D}_{T} \mathbf{B}^{\prime}\right\|_{\mathrm{MAX}} \\
& +\left\|\mathbf{C}_{T} \operatorname{cov}\left(\mathbf{f}_{t}\right) \mathbf{C}_{T}^{\prime}\right\|_{\mathrm{MAX}}+\left\|2 \mathbf{B D}_{T} \mathbf{C}_{T}^{\prime}\right\|_{\mathrm{MAX}} \\
& +\left\|\mathbf{C}_{T} \mathbf{D}_{T} \mathbf{C}_{T}^{\prime}\right\|_{\mathrm{MAX}}+\left\|\widehat{\boldsymbol{\Sigma}}_{u}^{\mathcal{T}}-\boldsymbol{\Sigma}_{u}\right\|_{\mathrm{MAX}} .
\end{aligned}
$$


By assumption, both $\|\mathbf{B}\|_{\text {MAX }}$ and $\left\|\operatorname{cov}\left(\mathbf{f}_{t}\right)\right\|_{\text {MAX }}$ are bounded uniformly in $(p, K, T)$. In addition, let $\mathbf{e}_{i}$ be a $p$-dimensional column vector whose $i$ th component is one with the remaining components being zeros. Then under the events $\left\|\mathbf{D}_{T}\right\|_{\mathrm{MAX}} \leq C \sqrt{(\log T) / T}, \max _{i \leq K, j \leq p}\left|\frac{1}{T} \sum_{t=1}^{T} f_{i t} u_{j t}\right| \leq C \sqrt{(\log p) / T}$, $\left\|\frac{1}{T} \mathbf{X} \mathbf{X}^{\prime}\right\| \leq C$, and $\max _{i \leq p}\left\|\widehat{\mathbf{b}}_{i}-\mathbf{b}_{i}\right\| \leq C \sqrt{K(\log p) / T}$, we have, for some $C^{\prime}>0$,

$\left\|2 \mathbf{C}_{T} \operatorname{cov}\left(\mathbf{f}_{t}\right) \mathbf{B}^{\prime}\right\|_{\mathrm{MAX}} \leq 2 \max _{i, j \leq p}\left\|\mathbf{e}_{i}^{\prime} \mathbf{C}_{T} \operatorname{cov}\left(\mathbf{f}_{t}\right) \mathbf{B}^{\prime} \mathbf{e}_{j}\right\|$

$$
\begin{aligned}
& \leq 2 \max _{i \leq p}\left\|\widehat{\mathbf{b}}_{i}-\mathbf{b}_{i}\right\|\left\|\operatorname{cov}\left(\mathbf{f}_{t}\right)\right\| \max _{j \leq p}\left\|\mathbf{b}_{j}\right\| \\
& \leq C^{\prime} K \sqrt{\frac{\log p}{T}} \\
\left\|\mathbf{C}_{T}\right\|_{\mathrm{MAX}} & =\max _{i, j \leq p}\left|\mathbf{e}_{i}^{\prime} \frac{1}{T} \mathbf{E} \mathbf{X}^{\prime}\left(\frac{1}{T} \mathbf{X} \mathbf{X}^{\prime}\right)^{-1} \mathbf{e}_{j}\right| \\
& \leq \max _{i \leq p}\left\|\mathbf{e}_{i}^{\prime} \frac{1}{T} \mathbf{E} \mathbf{X}^{\prime}\right\| \cdot\left\|\left(\frac{1}{T} \mathbf{X} \mathbf{X}^{\prime}\right)^{-1}\right\| \\
& \leq \sqrt{K} \max _{i \leq K, j \leq p}\left|\frac{1}{T} \sum_{t=1}^{T} f_{i t} u_{j t}\right| \cdot\left\|\left(\frac{1}{T} \mathbf{X} \mathbf{X}^{\prime}\right)^{-1}\right\| \\
& \leq C^{\prime} \sqrt{K(\log p) / T},
\end{aligned}
$$

$$
\left\|\mathbf{B D}_{T} \mathbf{B}^{\prime}\right\|_{\mathrm{MAX}} \leq K^{2}\|\mathbf{B}\|_{\mathrm{MAX}}^{2}\left\|\mathbf{D}_{T}\right\|_{\mathrm{MAX}} \leq C^{\prime} K^{2} \sqrt{\frac{\log T}{T}}
$$$$
\left\|\mathbf{C}_{T} \operatorname{cov}\left(\mathbf{f}_{t}\right) \mathbf{C}_{T}^{\prime}\right\|_{\mathrm{MAX}} \leq \max _{i, j \leq p}\left\|\mathbf{e}_{i}^{\prime} \mathbf{C}_{T} \operatorname{cov}\left(\mathbf{f}_{t}\right) \mathbf{C}_{T}^{\prime} \mathbf{e}_{j}\right\|
$$

$$
\begin{aligned}
& \leq \max _{i \leq p}\left\|\mathbf{e}_{i}^{\prime} \mathbf{C}_{T}\right\|^{2}\left\|\operatorname{cov}\left(\mathbf{f}_{t}\right)\right\| \\
& \leq \frac{C^{\prime} K^{2} \log p}{T},
\end{aligned}
$$

$$
\left\|2 \mathbf{B D}_{T} \mathbf{C}_{T}^{\prime}\right\|_{\mathrm{MAX}} \leq 2 K^{2}\|\mathbf{B}\|_{\mathrm{MAX}}\left\|\mathbf{D}_{T}\right\|_{\mathrm{MAX}}\left\|\mathbf{C}_{T}\right\|_{\mathrm{MAX}}
$$

$$
=o\left(K^{2} \sqrt{\frac{\log T}{T}}\right)
$$

and

(B.18) $\left\|\mathbf{C}_{T} \mathbf{D}_{T} \mathbf{C}_{T}^{\prime}\right\|_{\mathrm{MAX}} \leq K^{2}\left\|\mathbf{D}_{T}\right\|_{\mathrm{MAX}}\left\|\mathbf{C}_{T}\right\|_{\mathrm{MAX}}^{2}=o\left(K^{2} \sqrt{\frac{\log T}{T}}\right)$. 
Moreover, the $(i, j)$ th entry of $\widehat{\boldsymbol{\Sigma}}_{u}^{\mathcal{T}}-\boldsymbol{\Sigma}_{u}$ is given by

$$
\widehat{\sigma}_{i j} I\left(\left|\widehat{\sigma}_{i j}\right| \geq \omega_{T} \sqrt{\widehat{\theta}_{i j}}\right)-\sigma_{i j}= \begin{cases}-\sigma_{i j}, & \text { if }\left|\widehat{\sigma}_{i j}\right|<\omega_{T} \sqrt{\widehat{\theta}_{i j}}, \\ \widehat{\sigma}_{i j}-\sigma_{i j}, & \text { o.w. }\end{cases}
$$

Hence $\left\|\widehat{\boldsymbol{\Sigma}}_{u}^{\mathcal{T}}-\boldsymbol{\Sigma}_{u}\right\|_{\mathrm{MAX}} \leq \max _{i, j \leq p}\left|\sigma_{i j}-\widehat{\sigma}_{i j}\right|+\omega_{T} \max _{i, j \leq p} \sqrt{\widehat{\theta}_{i j}}$, which implies that with probability at least $1-O\left(p^{-2}+T^{-2}\right)$,

$$
\left\|\widehat{\Sigma}_{u}^{\mathcal{T}}-\Sigma_{u}\right\|_{\mathrm{MAX}} \leq C^{\prime} K \sqrt{\frac{\log p}{T}} .
$$

The result then follows from the combination of (B.12)-(B.19), (B.4) and Lemmas 3.1, B.1.

B.3. Proof of Theorem 3.2, part (ii). We first prove two technical lemmas to be used below.

LEMMA B.4. (i) $\lambda_{\min }\left(\mathbf{B}^{\prime} \boldsymbol{\Sigma}_{u}^{-1} \mathbf{B}\right) \geq c p$ for some $c>0$.

(ii) $\left\|\left[\operatorname{cov}(\mathbf{f})^{-1}+\mathbf{B}^{\prime} \Sigma_{u}^{-1} \mathbf{B}\right]^{-1}\right\|=O\left(p^{-1}\right)$.

PROOF. (i) We have

$$
\lambda_{\min }\left(\mathbf{B}^{\prime} \Sigma_{u}^{-1} \mathbf{B}\right) \geq \lambda_{\min }\left(\Sigma_{u}^{-1}\right) \lambda_{\min }\left(\mathbf{B}^{\prime} \mathbf{B}\right) .
$$

It then follows from Assumption 3.5 that $\lambda_{\min }\left(\mathbf{B}^{\prime} \mathbf{B}\right)>c p$ for some $c>0$ and all large $p$. The result follows since $\left\|\boldsymbol{\Sigma}_{u}\right\|$ is bounded away from infinity.

(ii) It follows immediately from

$$
\lambda_{\min }\left(\operatorname{cov}\left(\mathbf{f}_{t}\right)^{-1}+\mathbf{B}^{\prime} \Sigma_{u}^{-1} \mathbf{B}\right) \geq \lambda_{\min }\left(\mathbf{B}^{\prime} \Sigma_{u}^{-1} \mathbf{B}\right) .
$$

LEMMA B.5. There exists $C>0$ such that:

(i)

$$
P\left(\left\|\widehat{\mathbf{B}}^{\prime}\left(\widehat{\boldsymbol{\Sigma}}_{u}^{\mathcal{T}}\right)^{-1} \widehat{\mathbf{B}}-\mathbf{B}^{\prime} \boldsymbol{\Sigma}_{u}^{-1} \mathbf{B}\right\|>C p m_{T} K \sqrt{\frac{\log p}{T}}\right)=O\left(\frac{1}{p^{2}}+\frac{1}{T^{2}}\right) ;
$$

(ii)

$$
P\left(\left\|\left[\widehat{\operatorname{cov}}(\mathbf{f})^{-1}+\widehat{\mathbf{B}}^{\prime}\left(\widehat{\Sigma}_{u}^{\mathcal{T}}\right)^{-1} \widehat{\mathbf{B}}\right]^{-1}\right\|>\frac{C}{p}\right)=O\left(\frac{1}{p^{2}}+\frac{1}{T^{2}}\right)
$$

(iii) for $G=\left[\widehat{\operatorname{cov}}(\mathbf{f})^{-1}+\widehat{\mathbf{B}}^{\prime}\left(\widehat{\Sigma}_{u}^{\mathcal{T}}\right)^{-1} \widehat{\mathbf{B}}\right]^{-1}$,

$$
P\left(\left\|\widehat{\mathbf{B}} G \widehat{\mathbf{B}}^{\prime}\left(\widehat{\boldsymbol{\Sigma}}_{u}^{\mathcal{T}}\right)^{-1}\right\|>C\right)=O\left(\frac{1}{p^{2}}+\frac{1}{T^{2}}\right) .
$$


Proof. (i) Let $H=\left\|\widehat{\mathbf{B}}^{\prime}\left(\widehat{\boldsymbol{\Sigma}}_{u}^{\mathcal{T}}\right)^{-1} \widehat{\mathbf{B}}-\mathbf{B}^{\prime} \boldsymbol{\Sigma}_{u}^{-1} \mathbf{B}\right\|$.

$$
\begin{aligned}
H \leq & 2\left\|\mathbf{C}_{T}^{\prime} \boldsymbol{\Sigma}_{u}^{-1} \mathbf{B}\right\|+2\left\|\mathbf{C}_{T}^{\prime}\left(\left(\widehat{\boldsymbol{\Sigma}}_{u}^{\mathcal{T}}\right)^{-1}-\boldsymbol{\Sigma}_{u}^{-1}\right) \mathbf{B}\right\| \\
& +\left\|\mathbf{B}^{\prime \prime}\left(\left(\widehat{\boldsymbol{\Sigma}}_{u}^{\mathcal{T}}\right)^{-1}-\boldsymbol{\Sigma}_{u}^{-1}\right) \mathbf{B}\right\|+\left\|\mathbf{C}_{T}^{\prime} \boldsymbol{\Sigma}_{u}^{-1} \mathbf{C}_{T}\right\| \\
& +\left\|\mathbf{C}_{T}^{\prime}\left(\left(\widehat{\boldsymbol{\Sigma}}_{u}^{\mathcal{T}}\right)^{-1}-\boldsymbol{\Sigma}_{u}^{-1}\right) \mathbf{C}_{T}\right\| .
\end{aligned}
$$

The same argument of Fan, Fan and Lv (2008) (equation 14) implies that $\|\mathbf{B}\|_{F}=$ $O(\sqrt{p})$. Therefore, by Theorem 3.1 and Lemma B.2(ii), it is straightforward to verify the result.

(ii) Since $\left\|\mathbf{D}_{T}\right\|_{F} \geq\left\|\mathbf{D}_{T}\right\|$, according to Lemma B.2(i), there exists $C^{\prime}>0$ such that with probability ast least $1-O\left(T^{-2}\right),\left\|\mathbf{D}_{T}\right\|<C^{\prime} K \sqrt{(\log T) / T}$. Thus by Lemma A.1, for some $C^{\prime \prime}>0$,

$$
\begin{aligned}
P\left(\left\|\widehat{\operatorname{cov}}\left(\mathbf{f}_{t}\right)^{-1}-\operatorname{cov}\left(\mathbf{f}_{t}\right)^{-1}\right\|<C^{\prime \prime}\left\|\mathbf{D}_{T}\right\|\right) & \geq P\left(\left\|\mathbf{D}_{T}\right\|<C^{\prime} K \sqrt{\frac{\log T}{T}}\right) \\
& \geq 1-O\left(T^{-2}\right),
\end{aligned}
$$

which implies

$$
P\left(\left\|\widehat{\operatorname{cov}}\left(\mathbf{f}_{t}\right)^{-1}-\operatorname{cov}\left(\mathbf{f}_{t}\right)^{-1}\right\|<C^{\prime \prime} C^{\prime} K \sqrt{\frac{\log T}{T}}\right) \geq 1-O\left(T^{-2}\right) .
$$

Now let $\widehat{\mathbf{A}}=\widehat{\operatorname{cov}}\left(\mathbf{f}_{t}\right)^{-1}+\widehat{\mathbf{B}}^{\prime}\left(\widehat{\boldsymbol{\Sigma}}_{u}^{\mathcal{T}}\right)^{-1} \widehat{\mathbf{B}}$, and $\mathbf{A}=\operatorname{cov}\left(\mathbf{f}_{t}\right)^{-1}+\mathbf{B}^{\prime} \boldsymbol{\Sigma}_{u}^{-1} \mathbf{B}$. Then part (i) and (B.20) imply

$$
P\left(\|\widehat{\mathbf{A}}-\mathbf{A}\|<C^{\prime \prime} C^{\prime} K \sqrt{\frac{\log T}{T}}+C p m_{T} K \sqrt{\frac{\log p}{T}}\right)
$$

$$
\geq 1-O\left(\frac{1}{p^{2}}+\frac{1}{T^{2}}\right) .
$$

In addition, $m_{T} K \sqrt{(\log p) / T}=o(1)$. Hence by Lemmas A.1, B.4(ii), for some $C>0$,

$$
P\left(\lambda_{\min }(\widehat{\mathbf{A}}) \geq C p\right) \geq P(\|\widehat{\mathbf{A}}-\mathbf{A}\|<C p) \geq 1-O\left(\frac{1}{p^{2}}+\frac{1}{T^{2}}\right),
$$

which implies the desired result.

(iii) By the triangular inequality, $\|\widehat{\mathbf{B}}\|_{F} \leq\left\|\mathbf{C}_{T}\right\|_{F}+O(\sqrt{p})$. Hence Lemma B.2(ii) implies, for some $C>0$,

$$
P\left(\|\widehat{\mathbf{B}}\|_{F} \leq C \sqrt{p}\right) \geq 1-O\left(T^{-2}+p^{-2}\right) .
$$

In addition, since $\left\|\Sigma_{u}^{-1}\right\|$ is bounded, it then follows from Theorem 3.1 that $\left\|\left(\widehat{\Sigma}_{u}^{\mathcal{T}}\right)^{-1}\right\|$ is bounded with probability at least $1-O\left(p^{-2}+T^{-2}\right)$. The result then follows from the fact that

$$
P\left(\|G\|>C p^{-1}\right)=O\left(\frac{1}{p^{2}}+\frac{1}{T^{2}}\right),
$$


which is shown in part (ii).

To complete the proof of Theorem 3.2, part (ii), we follow similar lines of proof as in Fan, Fan and Lv (2008). Using the Sherman-Morrison-Woodbury formula, we have

$$
\begin{aligned}
\left\|\left(\widehat{\boldsymbol{\Sigma}}^{\mathcal{T}}\right)^{-1}-\boldsymbol{\Sigma}^{-1}\right\| \\
=\left\|\left(\widehat{\boldsymbol{\Sigma}}_{u}^{\mathcal{T}}\right)^{-1}-\boldsymbol{\Sigma}_{u}^{-1}\right\| \\
+\left\|\left(\left(\widehat{\boldsymbol{\Sigma}}_{u}^{\mathcal{T}}\right)^{-1}-\boldsymbol{\Sigma}_{u}^{-1}\right) \widehat{\mathbf{B}}\left[\widehat{\operatorname{cov}}(\mathbf{f})^{-1}+\widehat{\mathbf{B}}^{\prime}\left(\widehat{\boldsymbol{\Sigma}}_{u}^{\mathcal{T}}\right)^{-1} \widehat{\mathbf{B}}\right]^{-1} \widehat{\mathbf{B}}^{\prime}\left(\widehat{\boldsymbol{\Sigma}}_{u}^{\mathcal{T}}\right)^{-1}\right\| \\
+\left\|\left(\left(\widehat{\boldsymbol{\Sigma}}_{u}^{\mathcal{T}}\right)^{-1}-\boldsymbol{\Sigma}_{u}^{-1}\right) \widehat{\mathbf{B}}\left[\widehat{\operatorname{cov}}(\mathbf{f})^{-1}+\widehat{\mathbf{B}}^{\prime}\left(\widehat{\boldsymbol{\Sigma}}_{u}^{\mathcal{T}}\right)^{-1} \widehat{\mathbf{B}}\right]^{-1} \widehat{\mathbf{B}}^{\prime} \boldsymbol{\Sigma}_{u}^{-1}\right\| \\
+\left\|\boldsymbol{\Sigma}_{u}^{-1}(\widehat{\mathbf{B}}-\mathbf{B})\left[\widehat{\operatorname{cov}}(\mathbf{f})^{-1}+\widehat{\mathbf{B}}^{\prime}\left(\widehat{\boldsymbol{\Sigma}}_{u}^{\mathcal{T}}\right)^{-1} \widehat{\mathbf{B}}\right]^{-1} \widehat{\mathbf{B}}^{\prime} \boldsymbol{\Sigma}_{u}^{-1}\right\| \\
+\left\|\boldsymbol{\Sigma}_{u}^{-1}(\widehat{\mathbf{B}}-\mathbf{B})\left[\widehat{\operatorname{cov}}(\mathbf{f})^{-1}+\widehat{\mathbf{B}}^{\prime}\left(\widehat{\boldsymbol{\Sigma}}_{u}^{\mathcal{T}}\right)^{-1} \widehat{\mathbf{B}}\right]^{-1} \mathbf{B}^{\prime} \boldsymbol{\Sigma}_{u}^{-1}\right\| \\
+\| \boldsymbol{\Sigma}_{u}^{-1} \mathbf{B}\left(\left[\widehat{\operatorname{cov}}(\mathbf{f})^{-1}+\widehat{\mathbf{B}}^{\prime}\left(\widehat{\boldsymbol{\Sigma}}_{u}^{\mathcal{T}}\right)^{-1} \widehat{\mathbf{B}}\right]^{-1}\right. \\
\left.\quad-\left[\operatorname{cov}(\mathbf{f})^{-1}+\mathbf{B}^{\prime} \boldsymbol{\Sigma}_{u}^{-1} \mathbf{B}\right]^{-1}\right) \mathbf{B}^{\prime} \boldsymbol{\Sigma}_{u}^{-1} \| \\
=L_{1}+L_{2}+L_{3}+L_{4}+L_{5}+L_{6} .
\end{aligned}
$$

The bound of $L_{1}$ is given in Theorem 3.1.

For $G=\left[\widehat{\operatorname{cov}}(\mathbf{f})^{-1}+\widehat{\mathbf{B}}^{\prime}\left(\widehat{\Sigma}_{u}^{\mathcal{T}}\right)^{-1} \widehat{\mathbf{B}}\right]^{-1}$, then

$$
L_{2} \leq\left\|\left(\widehat{\boldsymbol{\Sigma}}_{u}^{\mathcal{T}}\right)^{-1}-\boldsymbol{\Sigma}_{u}^{-1}\right\| \cdot\left\|\widehat{\mathbf{B}} G \widehat{\mathbf{B}}^{\prime}\left(\widehat{\boldsymbol{\Sigma}}_{u}^{\mathcal{T}}\right)^{-1}\right\|
$$

It follows from Theorem 3.1 and Lemma B.5(iii) that

$$
P\left(L_{2} \leq C m_{T} K \sqrt{\frac{\log p}{T}}\right) \geq 1-O\left(\frac{1}{p^{2}}+\frac{1}{T^{2}}\right) .
$$

The same bound can be achieved in a same way for $L_{3}$. For $L_{4}$, we have

$$
L_{4} \leq\left\|\boldsymbol{\Sigma}_{u}^{-1}\right\|^{2} \cdot\|\widehat{\mathbf{B}}-\mathbf{B}\| \cdot\|\widehat{\mathbf{B}}\| \cdot\|G\| .
$$

It follows from Lemmas B.2, B.5(ii), and inequality (B.22) that

$$
P\left(L_{4} \leq C \sqrt{\frac{K \log p}{T}}\right) \geq 1-O\left(\frac{1}{p^{2}}+\frac{1}{T^{2}}\right) .
$$

The same bound also applies to $L_{5}$. Finally,

$$
L_{6} \leq\|\mathbf{B}\|^{2}\left\|\boldsymbol{\Sigma}_{u}^{-1}\right\|^{2}\left\|\widehat{\mathbf{A}}^{-1}-\mathbf{A}^{-1}\right\| \leq\|\mathbf{B}\|^{2}\left\|\boldsymbol{\Sigma}_{u}^{-1}\right\|^{2}\|\widehat{\mathbf{A}}-\mathbf{A}\| \cdot\left\|\widehat{\mathbf{A}}^{-1}\right\| \cdot\left\|\mathbf{A}^{-1}\right\|,
$$

where both $\widehat{\mathbf{A}}$ and $\mathbf{A}$ are defined after inequality (B.20). By Lemma B.4(ii), $\left\|\mathbf{A}^{-1}\right\|=O\left(p^{-1}\right)$. Lemma B.5(ii) implies $P\left(\left\|\widehat{\mathbf{A}}^{-1}\right\|>C p^{-1}\right)=O\left(p^{-2}+T^{-2}\right)$. Combining with (B.21), we obtain

$$
P\left(L_{6} \leq C m_{T} K \sqrt{\frac{\log p}{T}}\right) \geq 1-O\left(\frac{1}{p^{2}}+\frac{1}{T^{2}}\right) .
$$

The proof is completed by combining $L_{1} \sim L_{6}$. 


\section{APPENDIX C: PROOFS FOR SECTION 4}

The proof is similar to that of Lemma 3.1. Thus we sketch it very briefly. The OLS is given by

$$
\widehat{\mathbf{b}}_{i}=\left(\mathbf{X}_{i}^{\prime} \mathbf{X}_{i}\right)^{-1} \mathbf{X}_{i}^{\prime} \mathbf{y}_{i}, \quad i \leq p .
$$

The same arguments in the proof of Lemma B.1 can yield, for large enough $C>0$,

$$
P\left(\max _{i \leq p}\left\|\widehat{\mathbf{b}}_{i}-\mathbf{b}_{i}\right\|>C \sqrt{\frac{K \log p}{T}}\right)=O\left(\frac{1}{p^{2}}+\frac{1}{T^{2}}\right),
$$

which then implies the rate of

$$
\max _{i \leq p} \frac{1}{T} \sum_{t=1}^{T}\left(u_{i t}-\widehat{u}_{i t}\right)^{2} \leq \max _{i \leq p}\left\|\widehat{\mathbf{b}}_{i}-\mathbf{b}_{i}\right\|^{2} \frac{1}{T} \sum_{t=1}^{T}\left\|\mathbf{f}_{i t}\right\|^{2} .
$$

The result then follows from a straightforward application of Theorem 2.1.

\section{REFERENCES}

Antoniadis, A. and Fan, J. (2001). Regularization of wavelet approximations. J. Amer. Statist. Assoc. 96 939-967. MR1946364

BAI, J. (2003). Inferential theory for factor models of large dimensions. Econometrica 71 135-171. MR1956857

BAI, J. and NG, S. (2002). Determining the number of factors in approximate factor models. Econometrica 70 191-221. MR1926259

BiCKel, P. J. and LEVINA, E. (2008a). Covariance regularization by thresholding. Ann. Statist. 36 2577-2604. MR2485008

BiCKEL, P. J. and LEVINA, E. (2008b). Regularized estimation of large covariance matrices. Ann. Statist. 36 199-227. MR2387969

CAI, T. and LIU, W. (2011). Adaptive thresholding for sparse covariance matrix estimation. J. Amer. Statist. Assoc. 106 672-684.

CAI, T. and ZHOU, H. (2010). Optimal rates of convergence for sparse covariance matrix estimation. Unpublished manuscript, Dept. Statistics, The Wharton School, Univ. Pennsylvania, Philadelphia, PA.

Chamberlain, G. and Rothschild, M. (1983). Arbitrage, factor structure and mean-variance analysis in large asset markets. Econometrica 51 1305-1324.

CONNOR, G. and KorAJCZYK, R. (1993). A Test for the number of factors in an approximate factor model. J. Finance 48 1263-1291.

FAMA, E. and FRENCH, K. (1992). The cross-section of expected stock returns. J. Finance $47427-$ 465.

FAN, J., FAN, Y. and LV, J. (2008). High dimensional covariance matrix estimation using a factor model. J. Econometrics 147 186-197. MR2472991

FAN, J., ZHANG, J. and YU, K. (2008). Asset allocation and risk assessment with gross exposure constraints for vast portfolios. Unpublished manuscript, Princeton Univ.

Gorman, M. (1981). Some Engel curves. In Essays in the Theory and Measurement of Consumer Behavior in Honor of Sir Richard Stone (A. Deaton, ed.). Cambridge Univ. Press, New York.

HARDING, M. (2009). Structural estimation of high-dimensional factor models. Unpublished manuscript, Stanford Univ. 
James, W. and Stein, C. (1961). Estimation with quadratic loss. In Proc. 4th Berkeley Sympos. Math. Statist. and Prob., Vol. I 361-379. Univ. California Press, Berkeley, CA. MR0133191

KMEntA, J. and Gilbert, R. (1970). Estimation of seemingly unrelated regressions with autoregressive disturbances. J. Amer. Statist. Assoc. 65 186-196.

LAM, C. and FAN, J. (2009). Sparsistency and rates of convergence in large covariance matrix estimation. Ann. Statist. 37 4254-4278. MR2572459

LEWBEL, A. (1991). The rank of demand systems: Theory and nonparametric estimation. Econometrica 59 711-730.

Merlevède, F., Peligrad, M. and Rio, E. (2009). A Bernstein type inequality and moderate deviations for weakly dependent sequences. Unpublished manuscript, Univ. Paris Est.

Rothman, A. J., LeVINA, E. and ZHU, J. (2009). Generalized thresholding of large covariance matrices. J. Amer. Statist. Assoc. 104 177-186. MR2504372

ZELLNER, A. (1962). An efficient method of estimating seemingly unrelated regressions and tests for aggregation bias. J. Amer. Statist. Assoc. 57 348-368. MR0139235

DEPARTMENT OF OPERATIONS RESEARCH AND FINANCIAL ENGINEERING

PRINCETON UNIVERSITY

PRINCETON, NEW JERSEY 08544

USA

E-MAIL: jqfan@princeton.edu yuanliao@princeton.edu mincheva@princeton.edu 